\title{
Neutralization mechanism of human monoclonal antibodies against Rift Valley fever virus
}

\author{
Qihui Wang $\oplus^{1,2,3,21}$, Tong Ma $\oplus^{1,4,21}$, Yan $\mathrm{Wu}^{2,5,21}$, Zhihai Chen $\oplus^{6,21}$, Hui Zeng7 , Zhou Tong1', Feng Gao8, \\ Jianxun Q ${ }^{9}$, Zhennan Zhao ${ }^{9}{ }^{9}$, Yan Chai ${ }^{9}$, Huabing Yang ${ }^{114}{ }^{14}$, Gary Wong ${ }^{10,11}$, Yuhai Bi ${ }^{9}$, Lili Wu $\odot 1$, \\ Rui Shi ${ }^{1}$, Mi Yang ${ }^{1}$, Jian Song ${ }^{9}{ }^{9}$, Haihai Jiang ${ }^{9,12}$, Zhiqiang An ${ }^{3}$, Junzhi Wang ${ }^{13}$, Tilahun D. Yilma ${ }^{14,15}$, \\ YiShi ${ }^{22,9,16}$, William J.Liu" ${ }^{17}$, MifangLiang ${ }^{17}$, Chuan Qin ${ }^{18}$, GeorgeF. Gao ${ }^{2,5,9,12,16,17,19 \star}$ and Jinghua Yan ${ }^{1,2,4,9,20 \star}$
}

Rift Valley fever virus (RVFV) is a mosquito-borne pathogen that causes substantial morbidity and mortality in livestock and humans. To date, there are no licensed human vaccines or therapeutics available. Here, we report the isolation of monoclonal antibodies from a convalescent patient, targeting the RVFV envelope proteins $\mathrm{Gn}$ and Gc. The Gn-specific monoclonal antibodies exhibited much higher neutralizing activities in vitro and protection efficacies in mice against RVFV infection, compared to the Gc-specific monoclonal antibodies. The $\mathrm{Gn}$ monoclonal antibodies were found to interfere with soluble $\mathrm{Gn}$ binding to cells and prevent infection by blocking the attachment of virions to host cells. Structural analysis of $\mathrm{Gn}$ complexed with four Gn-specific monoclonal antibodies resulted in the definition of three antigenic patches (A, B and C) on Gn domain I. Both patches $A$ and $B$ are major neutralizing epitopes. Our results highlight the potential of antibody-based therapeutics and provide a structure-based rationale for designing vaccines against RVFV.

R ift Valley fever virus (RVFV), the causative agent of zoonotic Rift Valley fever (RVF), is one of the most serious arbovirus threats to both human and animal health. Since its identification in $1931^{1}$, the periodical RVF epizootic has infected millions of ruminants and resulted in major losses in the livestock industry ${ }^{2,3}$. Meanwhile, from 2000 to June 2018, 4,830 cases of severe RVF in humans were reported to the World Health Organization, including 967 related deaths, with a case fatality rate of approximately $20.0 \%{ }^{4}$. Although formerly restricted to circulation in the African continent, RVFV spread, causing an epidemic in the Kingdom of Saudi Arabia and Yemen in $2000^{5}$. In 2016, China reported its first imported case, a patient returning from Angola, a non-epidemic country ${ }^{6}$. Many strategies for RVFV vaccines have been developed since the virus was first characterized ${ }^{7}$. However, in the 70 years since its characterization, no licensed vaccine preparations nor therapeutics have been approved for use in humans ${ }^{7}$. Therefore, effective antivirals are urgently needed.

RVFV belongs to the genus Phlebovirus under the family Phenuiviridae in the order Bunyavirales ${ }^{8}$. The virus, a mosquito- borne arbovirus, contains a tripartite RNA genome, consisting of large, medium (M) and small segments ${ }^{9}$. Two glycoproteins on the viral envelope, Gn and Gc, which are important components for virus entry and membrane fusion ${ }^{10-12}$, are encoded by the $M$ segment. The structure of RVFV Gn exhibits similar topology as its counterpart of severe fever with thrombocytopenia syndrome virus (SFTSV), another Phlebovirus member ${ }^{13,14}$. It is presumed that, on the RVFV virion in its pre-fusion state, Gc lines the inner half of the glycoprotein shell and forms an interface with Gn domain II (DII). The Gc fusion loop is shielded from solvents by Gn DI and DII, both of which are externally exposed ${ }^{13-15}$. Considerable data from vaccine studies suggest that neutralizing antibodies elicited by vaccines containing $\mathrm{Gn}$ alone or in combination with Gc are sufficient to confer protection against RVFV challenge in mice and livestock ${ }^{16-21}$.

Here, we isolated eight Gn-specific monoclonal antibodies and one Gc-specific monoclonal antibody from a convalescent RVF patient. The results showed that $\mathrm{Gn}$ monoclonal antibodies blocked the binding of $\mathrm{Gn}$ on the virions to susceptible cells, thus neutralizing viral infection. Additional structural analysis of $\mathrm{Gn}$ complexed

'CAS Key Laboratory of Microbial Physiological and Metabolic Engineering, Institute of Microbiology, Chinese Academy of Sciences, Beijing, China. ${ }^{2}$ Shenzhen Key Laboratory of Pathogen and Immunity, Shenzhen Third People's Hospital, Shenzhen, China. ${ }^{3}$ Texas Therapeutics Institute, Brown Foundation Institute of Molecular Medicine, University of Texas Health Science Center at Houston, Houston, TX, USA. ${ }^{4}$ Institute of Physical Science and Information, Anhui University, Hefei, China. ${ }^{5}$ Research Network of Immunity and Health (RNIH), Beijing Institutes of Life Science, Chinese Academy of Sciences, Beijing, China. ${ }^{6}$ Center of Infectious Disease, Beijing Ditan Hospital, Capital Medical University, Beijing, China. ${ }^{7}$ Beijing Key Laboratory of Emerging Infectious Diseases, Institute of Infectious Diseases, Beijing Ditan Hospital, Capital Medical University, Beijing, China. ${ }^{8}$ Institute of Genetics and Developmental Biology, Chinese Academy of Sciences, Beijing, China. ${ }^{9}$ CAS Key Laboratory of Pathogenic Microbiology and Immunology, Institute of Microbiology, Chinese Academy of Sciences, Beijing, China. ${ }^{10}$ Département de Microbiologie-Infectiologie et d'Immunologie, Université Laval, Quebec, Quebec, Canada. "Institut Pasteur of Shanghai, Chinese Academy of Sciences, Shanghai, China. ${ }^{12}$ College of Veterinary Medicine, China Agricultural University, Beijing, China. ${ }^{13}$ Key Laboratory of the Ministry of Health for Research on Quality and Standardization of Biotech Products, National Institutes for Food and Drug Control, Beijing, China. ${ }^{14}$ International Laboratory of Molecular Biology for Tropical Disease Agents, School of Veterinary Medicine, University of California, Davis, Davis, CA, USA. ${ }^{15}$ Department of Medical Microbiology and Immunology, School of Medicine, University of California, Davis, Davis, CA, USA. ${ }^{16}$ Savaid Medical School, University of Chinese Academy of Sciences, Beijing, China. ${ }^{17}$ National Institute for Viral Disease Control and Prevention, Chinese Center for Disease Control and Prevention (China CDC), Beijing, China. ${ }^{18}$ Key Laboratory of Human Diseases Comparative Medicine, Ministry of Health, Institute of Medical Laboratory Animal Science, Chinese Academy of Medical Sciences, Beijing, China. ${ }^{19}$ Collaborative Innovation Center for Diagnosis and Treatment of Infectious Diseases, Zhejiang University, Hangzhou, China. ${ }^{20}$ College of Life Science, University of Chinese Academy of Sciences, Beijing, China. ${ }^{21}$ These authors contributed equally: Qihui Wang, Tong Ma, Yan Wu, Zhihai Chen. ${ }^{\star}$ e-mail: gaof@im.ac.cn; yanjh@im.ac.cn 
with monoclonal antibodies revealed two neutralizing hotspots, targeted by most of the isolated $\mathrm{Gn}$ monoclonal antibodies. Our results strongly indicate that human infection by RVFV stimulates a robust humoral immune response, and suggest the high potential of antibody-based therapeutics. Moreover, the structural studies presented here shed light on the assembly of virions and provide a structurebased rationale for the design of future RVFV-specific vaccines.

\section{Results}

Antibody isolation and binding characterization. We applied both soluble $\mathrm{Gn}$ and $\mathrm{Gc}$ as baits to isolate specific memory B cells from an RVF convalescent plasma ${ }^{6}$, which displayed a strong reaction to both Gn and Gc (Supplementary Figs. 1 and 2). Then, the variable regions of IgG antibodies from each cell were determined (Supplementary Table 1). The nine monoclonal antibodies exhibited low somatic hypermutation (SHM) rates compared to their germline genes, with nucleotide identities higher than $97 \%$ for the variable regions in both the heavy chain $\left(\mathrm{V}_{\mathrm{H}}\right)$ and the light chain $\left(\mathrm{V}_{\mathrm{L}}\right)$. Additionally, both R13 and R17 incorporated the $\mathrm{V}_{\mathrm{L}}$ identical to their germline ancestors. R22 was a germline monoclonal antibody without SHM mutations. Interestingly, two pairs of monoclonal antibodies (R12 and R13; R16 and R17) incorporated light chains (L) with the same V and J alleles (Supplementary Tables 1 and 2). Next, each monoclonal antibody was converted to an IgG1 isotype for further experiments.

Via fluorescence-activated cell sorting (FACS), nine antibodies bound to cells expressing the M gene (Fig. 1a). However, when Gn and Gc were separated, eight monoclonal antibodies, namely R4, R12, R13, R15, R16, R17, R19 and R22, exclusively interacted with cells containing Gn. Only R5 bound to Gc-expressing cells (Fig. 1a). Consistently, the monoclonal antibodies displayed interactions with either Gn or Gc when assessed by surface plasmon resonance (SPR). The binding affinities between $\mathrm{Gn}$ and monoclonal antibodies varied, ranging from tens (R15, R22, R19 and R12) to hundreds of nanomolar (R4, R13, R16 and R17). The Gc monoclonal antibody R5 manifested a $K_{\mathrm{d}}$ of approximately $120 \mathrm{nM}$ (Fig. $1 \mathrm{~b}$ and Supplementary Fig. 3). No binding was observed between the isolated monoclonal antibodies with the counterparts in SFTSV (Fig. 1b).

Neutralization activities in vitro and protection efficacies in vivo against RVFV infection. Using a previously reported FACS-based assay with Vero cells ${ }^{22}$, the Gn monoclonal antibodies exhibited much more intense neutralizing potencies than the Gc monoclonal antibody R5 (Fig. 2 and Supplementary Data Set). The median inhibitory concentration $\left(\mathrm{IC}_{50}\right)$ of $\mathrm{R} 5$ was calculated to be $1,370 \pm 530 \mathrm{ng} \mathrm{ml}^{-1}$. In contrast, R15 and R16 displayed high neutralizing activities, with $\mathrm{IC}_{50}$ at $0.53 \pm 0.25 \mathrm{ng} \mathrm{ml}^{-1}$ for R15 and $0.29 \pm 0.09 \mathrm{ng} \mathrm{ml}^{-1}$ for R16 (Fig. 2c). The $\mathrm{IC}_{50}$ values of both $\mathrm{R} 12$ and $\mathrm{R} 17$ increased to several nanograms per millilitre (Fig. 2d). R4, R13, R19 and R22 displayed values in the range of tens of nanograms per millilitre (Fig. 2e).

The protection efficacies of monoclonal antibodies against RVFV infection were evaluated in a mouse model ${ }^{23}$ (Supplementary Fig. 4). In the post-exposure setting, mice challenged with RVFV died within 5 days post-infection. All mice in the eight $\mathrm{Gn}$ monoclonalantibody-treated groups survived, except for one, injected with R22 $(n=4)$, which died at 11 days post-infection (Fig. 2f). Compared with the PBS group, Gn monoclonal-antibody-treated mice displayed similar body weight increases over the 14 days of observation (Fig. 2g). In contrast, in the Gc monoclonal antibody R5-treated group, only one survived ( $n=5$, Fig. $2 \mathrm{f})$. Similarly, Gn monoclonal antibodies prevented RVFV infection in the pre-exposure setting. All Gn monoclonal-antibody-treated mice survived, whereas four mice injected with R5 died ( $n=5$, Fig. 2 h,i).

Neutralizing mechanisms of Gn monoclonal antibodies. Viral attachment to susceptible cells is the initial step of viral infection ${ }^{24,25}$.
We prepared a Gn tetramer to amplify the interaction signals and used them to stain Huh7 cells ${ }^{26}$. Pre-incubation of the Gn tetramer with the Gn monoclonal antibodies, not the Gc monoclonal antibody R5, before staining Huh7 cells can prevent fluorescence shift (Fig. 3a). Following a reported procedure with some modifications ${ }^{27}$ (Supplementary Fig. 5), we found that all RVFV monoclonal antibodies associated with both Huh7 and Vero cells, if pre-incubated with the virus (Supplementary Fig. 6), suggesting that all RVFV monoclonal antibodies could bind to the virions. Compared with cells incubated with RVFV and tested with the indicated monoclonal antibody (marked as Virus binding in Fig. 3b), pre-incubation of the virus with each $\mathrm{Gn}$ monoclonal antibody before binding to Vero cells (marked as Indicated monoclonal antibody in Fig. $3 b)$ led to reduced fluorescence intensity, whereas the Gc monoclonal antibody R5 displayed no effect on the shift (Fig. 3b and Supplementary Fig. 5). In addition, the blocking effect of Gn monoclonal antibodies was observed in a concentration-dependent manner (Supplementary Fig. 7).

Molecular determinants of neutralizing monoclonal antibodies. To delineate the molecular basis of $\mathrm{Gn}$ monoclonal antibodies neutralizing RVFV infection, we solved the crystal structures of Gn complexed with the Fab form of the monoclonal antibodies R12, $\mathrm{R} 13$ and R15 and the scFv of R17. The resolutions for the complex structures are $2.7 \AA$ for R12, $2.0 \AA$ for R13, $2.4 \AA$ for R15, and $3.2 \AA$ for R17, respectively (Supplementary Table 3). Gn is composed of three domains, DI, DII and DIII ${ }^{13,14}$. Interestingly, each monoclonal antibody applies a similar perpendicular angle to interact with equal molar Gn (Fig. 4). In addition, all four monoclonal antibodies bind to DI, albeit R12 also interacts with Lys 395 in DIII (Supplementary Table 4). With the exception of complementaritydetermining region 1 (CDR1) in the $\mathrm{R} 13$ heavy chain (H), all CDRs from both heavy and light chains of R12, R13, R15 and R17 are involved in direct $\mathrm{Gn}$ interaction. In the four complexes, the buried surfaces on R12, R13, R15 and R17 are $672.3 \AA^{2}, 668.3 \AA^{2}, 622.7 \AA^{2}$ and $793.5 \AA^{2}$, respectively. Meanwhile, $G n$ is buried $703.1 \AA^{2}$, $719.6 \AA^{2}, 713 \AA^{2}$ and $830.3 \AA^{2}$ by the monoclonal antibodies R12, R13, R15 and R17, respectively.

Further scrutiny of the binding interface revealed that the epitopes of R12, R13 and R15 overlap substantially (Figs. 4 and 5a-i). $\mathrm{R} 12 \mathrm{H}$ forms a patch of electrostatic interactions with $\mathrm{Gn}$ residues in $\eta 2 \alpha 1$ (Asp 176) and residues adjacent to $\eta 2 \alpha 1$ (Lys 164, His 166, Tyr 168 and Lys 180). R12L interacts with Gn $\eta 2 \alpha 1$, with Gln 174 and Lys 294 in the centre (Fig. 5b,c). Consistently, a single mutated residue of either D176A, Q174A, K180A or K294A in Gn decreased or destroyed the association with R12 (Supplementary Fig. 8). Here, we designated the region including $\eta 2 \alpha 1\left({ }_{173}\right.$ TQEDATCK $\left._{180}\right)$ and $\mathrm{K} 294$ as antigenic patch A. Deleting antigenic site A (Gn- $\Delta \mathrm{A})$ abolished $\mathrm{Gn}$ binding by R12 (Fig. $6 a, b)$. Gn residues in the $\beta 3 / \beta 4$ loop $\left({ }_{271} \mathrm{CPPK}_{274}\right.$; named antigenic patch $\left.\mathrm{B}\right)$ also confer several van der Waals contacts with R12L (Supplementary Table 4).

$\mathrm{R} 13 \mathrm{~L}$ is the germline sequence of R12L (Supplementary Table 2). It adopts the same interaction mode as R12L to associate with Gn (Fig. 5c,f). Accordingly, the root-mean-square deviation between $\mathrm{Gn}$ with $\mathrm{R} 12 \mathrm{~L}$ and $\mathrm{R} 13 \mathrm{~L}$ reaches 0.388 (338 C $\alpha$ atoms). Compared with the germline R13L, R12L acquired Ser 31 through SHMs, which optimized its function (Supplementary Table 4 and Supplementary Fig. 9). Concomitantly, the long CDR3 (17 residues) in $\mathrm{R} 13 \mathrm{H}$ interacts hydrophilically with $\mathrm{Gn} \alpha 1$ helix $\left({ }_{176} \mathrm{DAT}_{178}\right)$, Lys 180 and Lys 294 (Fig. 5e). Introduction of either Q174A, D176A, $\mathrm{K} 180 \mathrm{~A}$ or K294A decreased the binding to the monoclonal antibody R13 (Supplementary Fig. 8). Gn- $\Delta$ A lost interaction with R13 (Fig. 6a,b).

R15, composed of two distinct chains, also targets antigenic patches A and B (Figs. 4 and $5 \mathrm{~g}-\mathrm{i}$ ). A single residue mutation in $\mathrm{Gn}$, such as Q174A, D176A, K180A or K294A, led to the varied loss of 

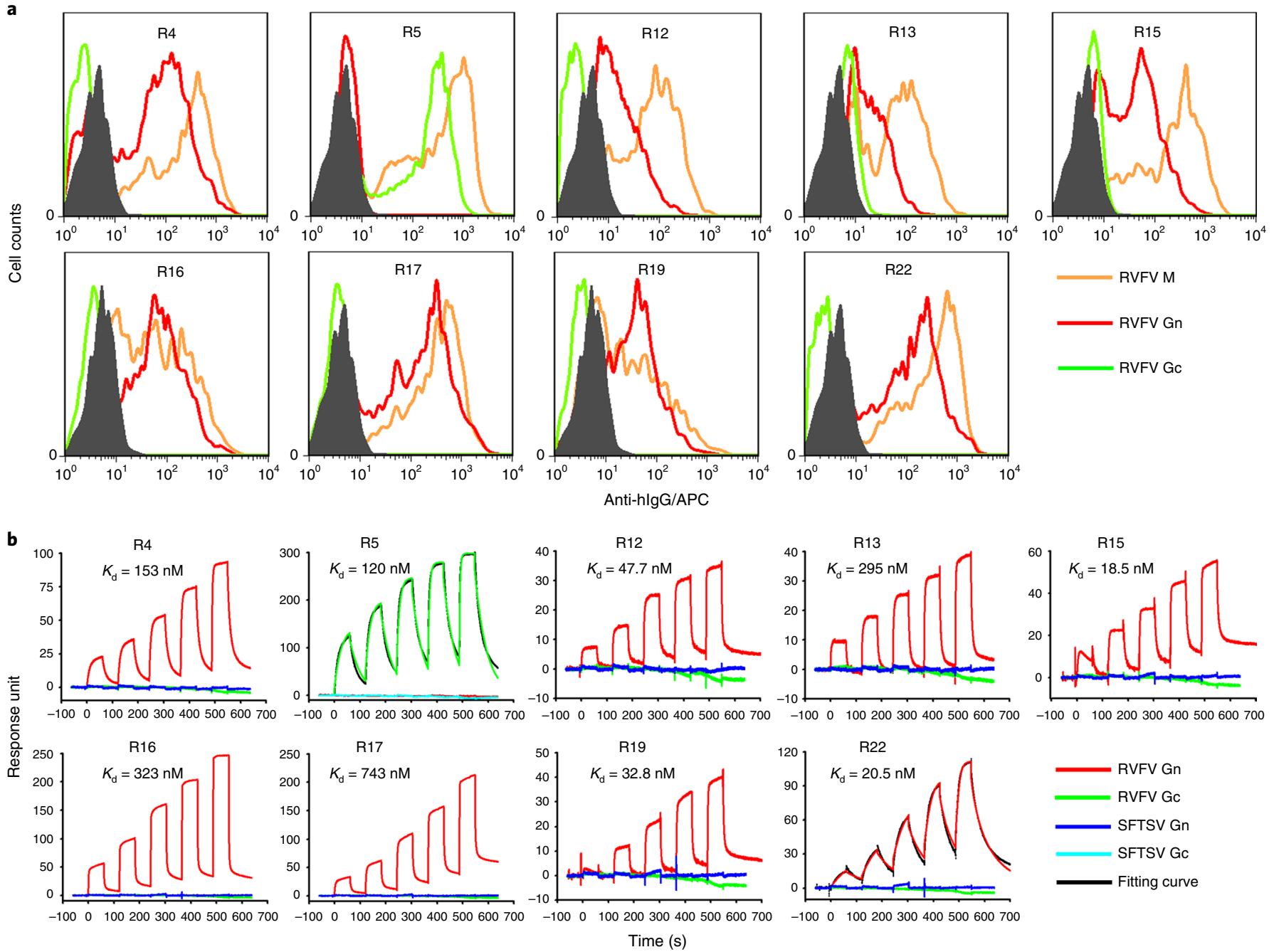

Fig. 1 | Binding characteristics of RVFV monoclonal antibodies. a, A flow cytometric assay of the indicated monoclonal antibodies involved in specific antigen binding. HEK293T cells were transfected with plasmids containing coding regions for eGFP-fused M (orange), Gn (red) and Gc (green), respectively, and then tested with the indicated monoclonal antibody. Cells transiently expressing M-eGFP stained with secondary antibodies (antihlgG/APC) were used as a negative control (black). b. An SPR assay characterizing the specific binding between the indicated monoclonal antibodies and specific antigens, including RVFV Gn (red), RVFV Gc (green), SFTSV Gn (blue) and SFTSV Gc (cyan). The black lines indicate the fitting curves. The calculated $K_{\mathrm{d}}$ for each monoclonal antibody binding to the specific antigen is listed below the name of the monoclonal antibody. The data are representative of three independent experiments.

binding ability to R15 (Supplementary Fig. 8). Gn- $\Delta$ A did not support R15 binding (Fig. 6a,b). Additionally, the introduction of both $\mathrm{P} 273 \mathrm{~A}$ and $\mathrm{K} 274 \mathrm{~A}(\mathrm{Gn}-\Delta \mathrm{B})$ to $\mathrm{Gn}$ also abolished binding to the monoclonal antibody R15 (Fig. 6a,b).

$\mathrm{R} 17$ mainly interacts with antigenic patch $\mathrm{B}$ and the $\beta 1$ strand, which was designated as antigenic patch $\mathrm{C}\left({ }_{223} \mathrm{KADPPSCD}_{230}\right)$ (Figs. 4 and $5 j-1)$. Through hydrophilic interactions, R17 anchors both terminal regions (Lys 223 and Asp 225 in the amino-terminal region, Asp 230 at the carboxy-terminal region) and the central region of the $\beta 1$ strand (Ser 228) (Fig. 5k). The Gn mutant with D225A completely lost its ability to bind to R17 (Supplementary Fig. 8). Simultaneously, R17 also interacts with antigenic patch B (Fig. 51) and the deletion of this region $(\mathrm{Gn}-\Delta \mathrm{B})$ destroyed binding to R17. R17 maintained association with Gn- $\Delta$ A (Fig. 6). HEK293T cells failed to express $\mathrm{Gn}$ without the $\beta 1$ strand, indicating its importance for the structural stability of Gn.

By docking the $\mathrm{Gn}$ monoclonal antibodies on the RVFV virions (PDB no. 6F9B, 6F9C and 6F9F) ${ }^{14}$, we found that all four monoclonal antibodies perpendicularly bind to the virions (Fig. $6 \mathrm{c}$ and
Supplementary Fig. 10). The shortest distances between monoclonal antibodies associating with adjacent Gns are $51.9 \AA$ for $\mathrm{R} 12,67.5 \AA$ for R13, and $40.3 \AA$ for R15, respectively. Notably, R17 $\mathrm{scFv}$, on certain positions of the virions, is indicated to sterically hinder the association of others (Supplementary Fig. 10d,e). R17 Fabs were simulated utilizing R12 Fabs and measured to be as short as approximately $20 \AA$ for the ones without clash on the virions (Supplementary Fig. 10).

Both antigenic patches $A$ and $B$ are hotspot neutralizing targets. We further tested the ability of the monoclonal antibodies without structural information to stain cells expressing different Gn mutants. R4 failed to bind to cells expressing Gn- $\Delta \mathrm{B}$. Although R4 maintained its interaction with Gn- $\Delta \mathrm{A}$, D176A or K180A decreased this association. Residues in patch $\mathrm{C}$ did not exert an effect on binding to R4. R16 could not bind to Gn- $\Delta \mathrm{A}$ or $\mathrm{Gn}-\Delta \mathrm{B}$, but normally interacted with Gn mutants in patch C. R19 associated with $\mathrm{Gn}-\Delta \mathrm{B}$, but not Gn- $\Delta \mathrm{A}$. Moreover, either D225A or D230A weakened the interaction. Although R22 could bind 

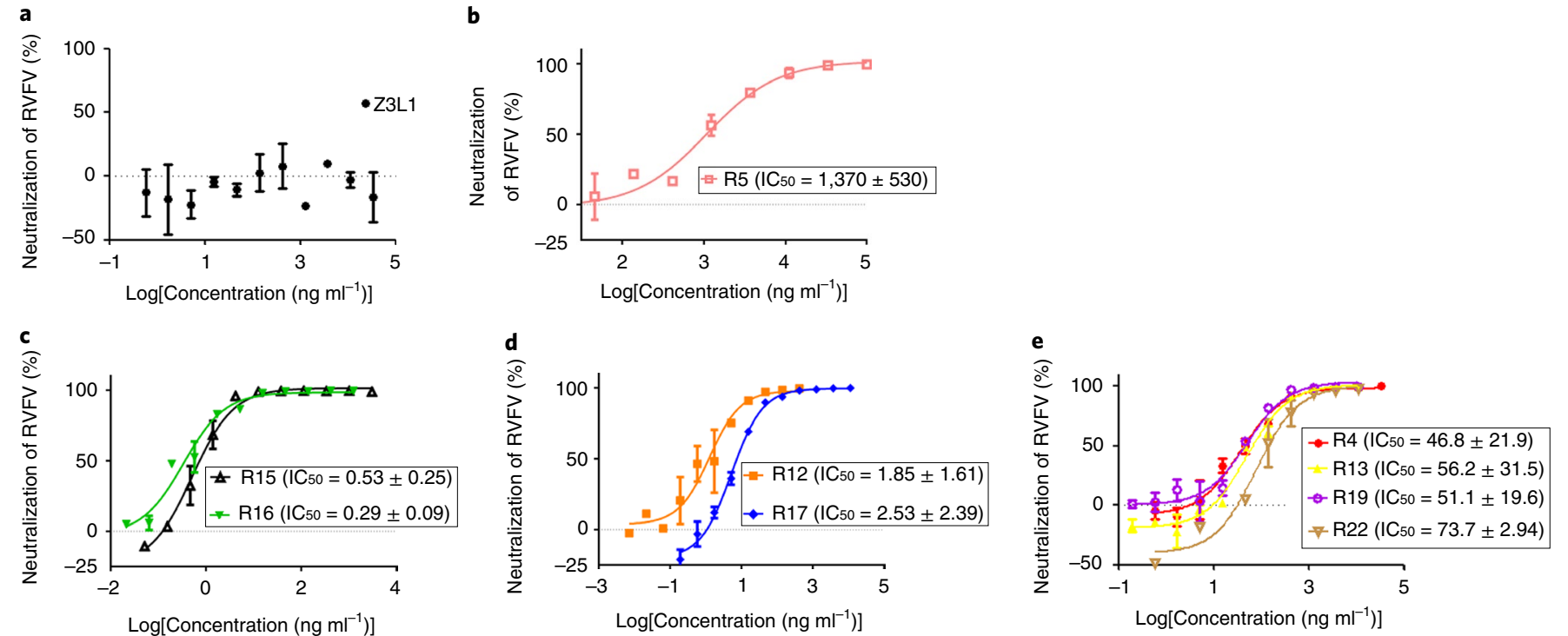

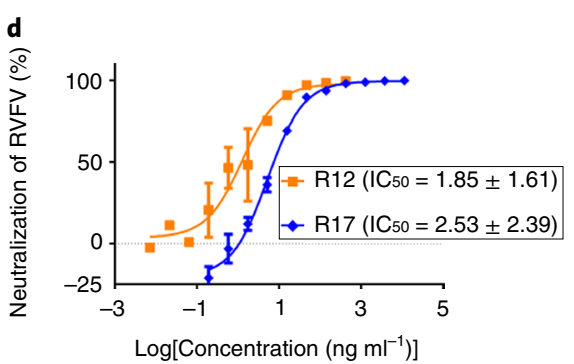

b
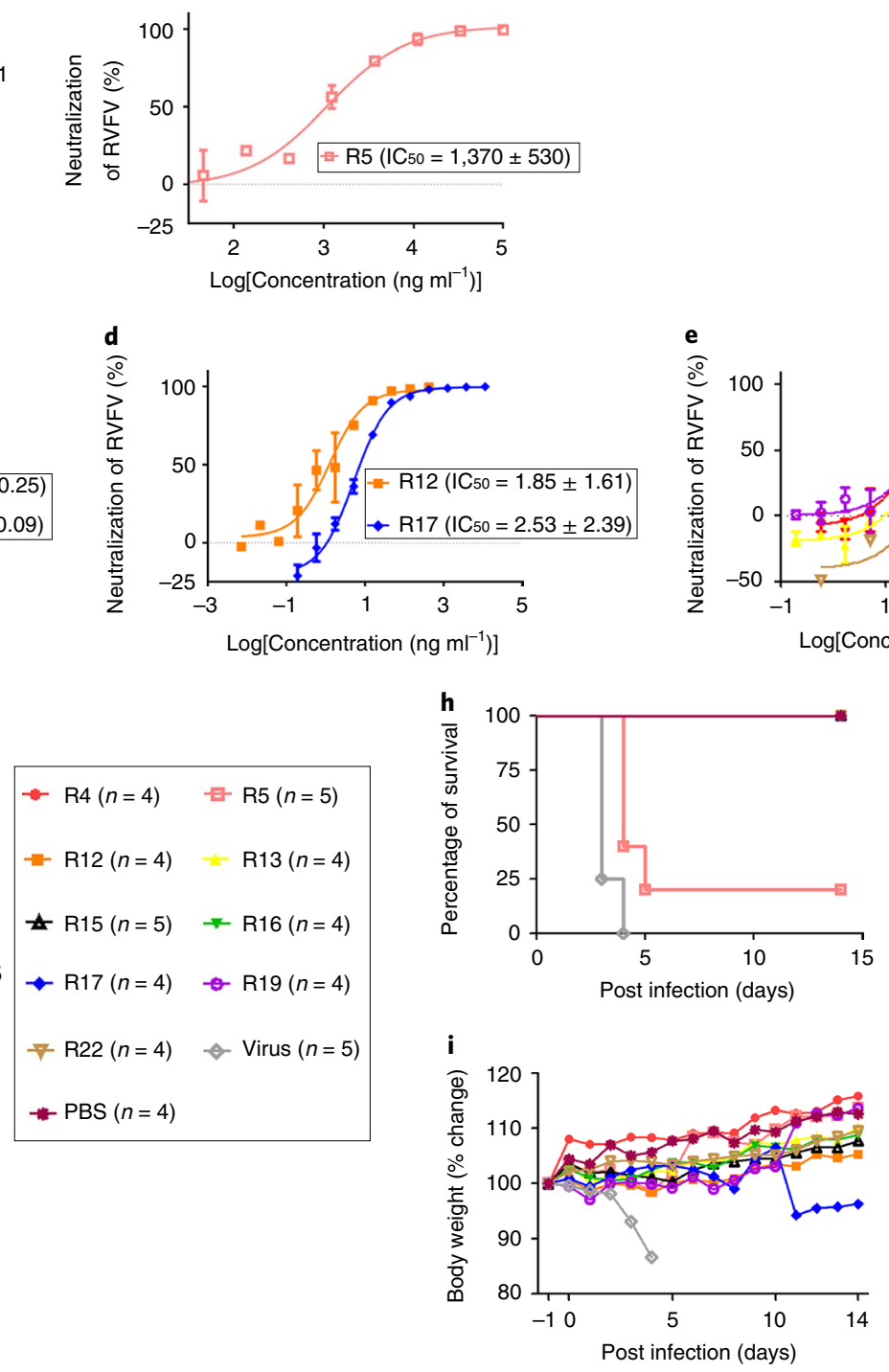

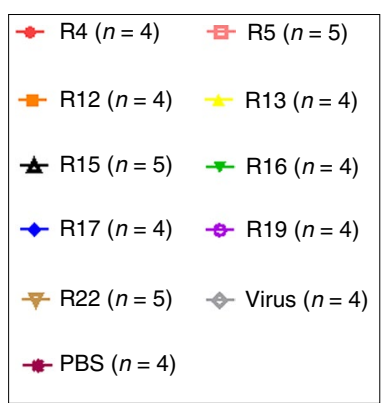

$\mathbf{g}$
ब
0
$\frac{0}{0}$
0
0
0
$\frac{1}{5}$
$\frac{0}{0}$
3
3े
0
0

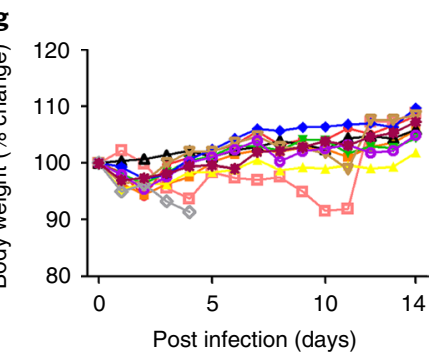

Fig. 2 | Protection potency of monoclonal antibodies against RVFV infection in vitro and in vivo. a-e, The neutralization activity of the indicated monoclonal antibody was evaluated by a FACS-based neutralization test using Vero cells. The number in parentheses following the name of the monoclonal antibody represents the mean \pm s.d. of the $\mathrm{IC}_{50}$ value $\left(\mathrm{ng} \mathrm{ml^{-1 }}\right)$ of $n=3$ independent experiments. The IC $\mathrm{C}_{50}$ was calculated using the equation of $\log$ [inhibitor] versus response in GraphPad Prism 5. The curves are representatives of three independent experiments. Zika monoclonal antibody Z3L1 was used as a negative control (a). RVFV monoclonal antibodies were grouped according to potency: IC $\mathbf{C}_{50}$ at micrograms per millilitre (b), sub-nanogram per millilitre (c), several nanograms per millilitre (d) and tens of nanograms per millilitre (e). f-i, For post-exposure treatment, BALB/c mice were challenged

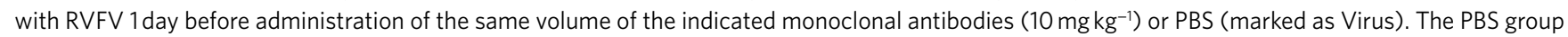
of mice were administered PBS instead of virus or monoclonal antibodies. Mouse survival (f) and body weight ( $\mathbf{g}$ ) were recorded daily. For pre-exposure treatment, mice were injected with the indicated monoclonal antibody $\left(10 \mathrm{mg} \mathrm{kg}^{-1}\right.$ ) or PBS (Virus), followed by challenge with RVFV 1 day later. Mice without inoculation with monoclonal antibodies or virus were marked as PBS. Mouse survival (h) and body weight (i) were recorded daily. Sample sizes of mice in both post-exposure (left panel) and pre-exposure (right panel) treatments are indicated. The survival curves were generated with a log-rank (Mantel-Cox) test using GraphPad Prism 5. Body weight loss curves were also generated using GraphPad Prism 5.

to both Gn- $\Delta \mathrm{A}$ and $\mathrm{Gn}-\Delta \mathrm{B}$, a single mutation of either D176A, K180A, D225A or D230A could reduce the level of interaction with R22 (Fig. 6 and Supplementary Fig. 8).

Thus, according to the critical patches that affect the Gn-monoclonal antibody interaction, the eight Gn monoclonal antibodies can be divided into four groups. Group 1 is that for which antigenic patch $\mathrm{A}$, not $\mathrm{B}$ or $\mathrm{C}$, is pivotal for the interaction, and contains R12, R13 and R19. For group 2 monoclonal antibodies, containing R15 and R16, both antigenic patches A and B, but not C, are critical for binding. For monoclonal antibodies in group 3, containing
$\mathrm{R} 4$ and $\mathrm{R} 17$, antigenic patch $\mathrm{B}$, not $\mathrm{A}$, plays a key role. In addition, antigenic patch $\mathrm{C}$ also determines the association with R17 (Fig. 6). The essential targets of R22, the only monoclonal antibody of group 4, require further study. Moreover, none of the Gn monoclonal antibodies or the Gc monoclonal antibody R5 could recognize the linearized epitopes, as indicated by western blot experiments (Supplementary Fig. 11).

Notably, residues that are equivalent to RVFV patches A, B and $\mathrm{C}$ among Phlebovirus are highly diverse (Supplementary Fig. 12). Further sequence analyses of 108 strains (see Methods) indicate that 

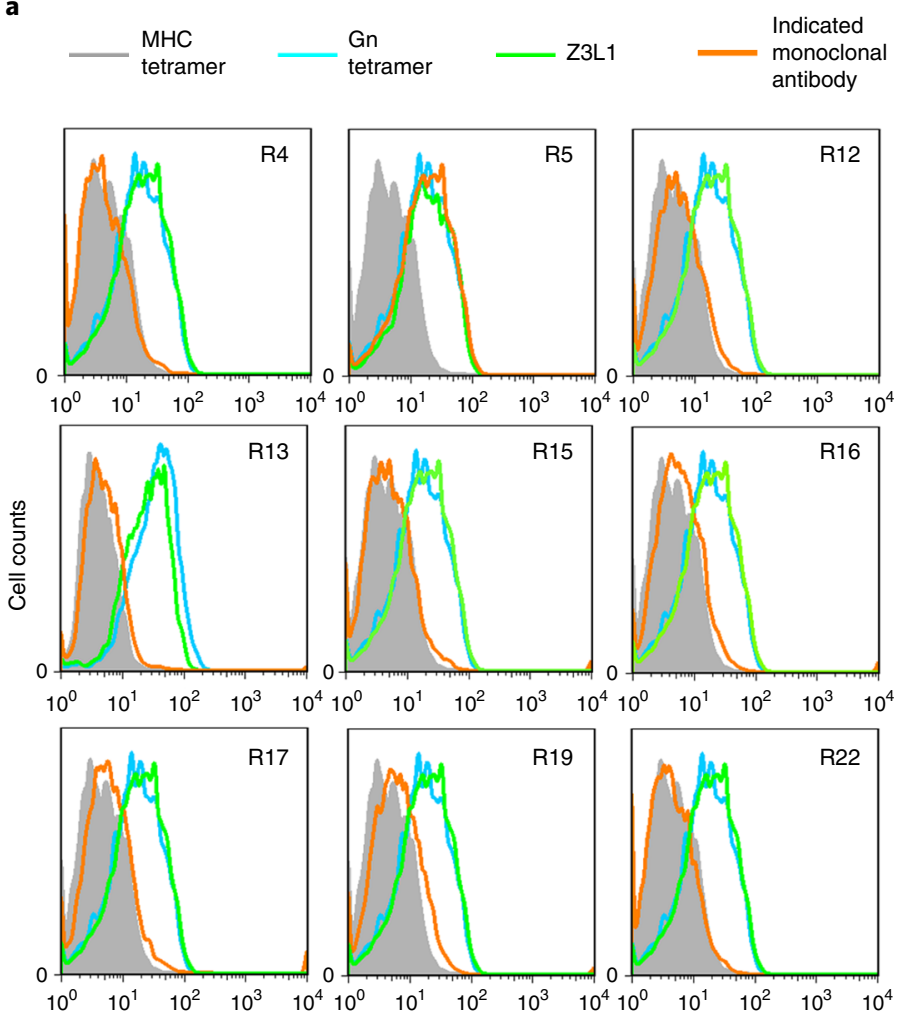

Tetramer/PE
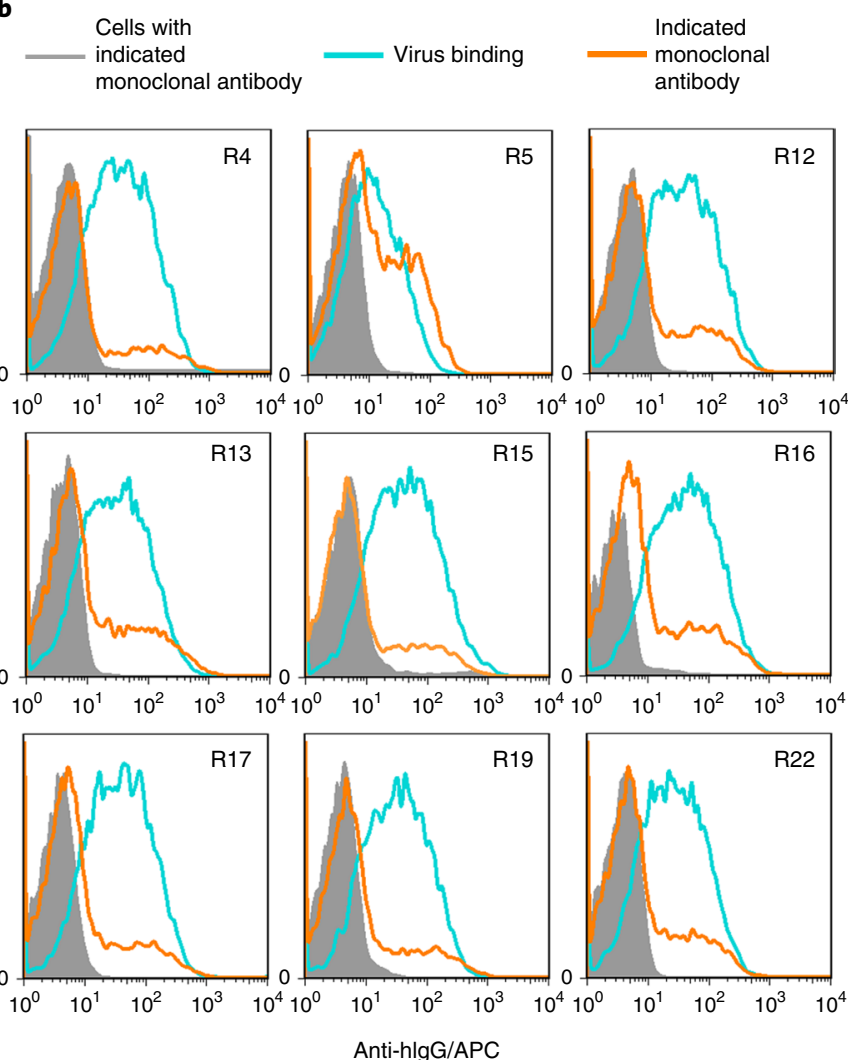

Anti-hlgG/APC

Fig. 3 Interference of Gn-cell and RVFV-cell interactions by Gn monoclonal antibodies. a, The Gn tetramer was prepared and tested by flow cytometry for its interaction with Huh7 cells, with or without the indicated RVFV monoclonal antibody. The MHC tetramer was used as a negative control for the Gn tetramer. Z3L1 was used as a negative control for the RVFV monoclonal antibodies. The data were processed with FlowJo software and are representative of two independent experiments. b, RVFV virions ( $\left.5 \times 10^{4} \mathrm{PFUs}\right)$ were incubated with Vero cells $\left(2 \times 10^{5}\right)$ for 30 min at $4^{\circ} \mathrm{C}$, and the bound virions were detected using the indicated RVFV monoclonal antibody $\left(200 \mu \mathrm{g} \mathrm{ml}^{-1}\right)$ by flow cytometry (cyan). For blocking evaluation, the virus was pre-incubated with the indicated monoclonal antibody at $37^{\circ} \mathrm{C}$ for 1 hour before its addition to Vero cells. The bound virions were then detected (orange). Cells interacting with the indicated monoclonal antibody and anti-hlgG/APC were used as negative controls to gate the cell (grey). The data are representative of two independent experiments.

residues constituting patch $\mathrm{B}$ are completely conserved. Three strains carry $\mathrm{T} 173 \mathrm{~L}^{28}$, and one strain carries $\mathrm{E} 175 \mathrm{G}^{29}$. Another strain carries K294E in patch A as well as D230N in patch C (Supplementary Fig. 13). Using flow cytometry analysis, Gn with T173L exerted no effect on binding to R17, but decreased or destroyed the association with other Gn monoclonal antibodies (Supplementary Fig. 14). Both E175G and K294E-D230N led to decreased binding to Gn monoclonal antibodies to various degrees (Supplementary Fig. 14).

Discussion and conclusion. In this study, we characterized human memory B cells encoding monoclonal antibodies targeting RVFV Gn and Gc. It seems that $\mathrm{Gn}$ is the immune-dominant antigen in human RVFV infections. Since the same primers were used to sequence genes in the isolated cells, there is little possibility that this discrepancy resulted from sequencing biases. However, we cannot exclude the possibility that the soluble Gc, which was used for screening, might exist in different conformations from the one that appears on the surface of RVFV ${ }^{14}$, and was thus missed by relevant Gc-targeting antibodies. Further studies are needed using labelled virus or virus-like particles to investigate the composition of Gn-specific and Gc-specific monoclonal antibodies in additional human patients. Among the nine monoclonal antibodies, seven incorporated $\lambda$ light chains. This might result from the different efficiencies of the primers used in PCR. Notably, similar $\lambda$ light chain-biased antibody responses were also reported in ferrets and humans infected with influenza virus ${ }^{30,31}$. Further studies are needed to determine whether this phenomenon is primer-dependent or case-dependent.

The isolated $\mathrm{Gn}$ monoclonal antibodies manifested relatively low binding affinities to $\mathrm{Gn}$. Consequently, complete blocking of the interaction between the Gn tetramer and Huh7 by these Gn monoclonal antibodies required that they be used in high concentrations. The relatively low SHM rates in the isolated monoclonal antibodies might explain their low binding affinities and imply that this infection was probably the primary infection in this patient. In fact, the patient was a temporary worker in Angola, but originated from China, where RVFV has not been recorded before. Despite having low maturation, Gn monoclonal antibodies demonstrate high neutralizing activities, preventing RVFV infection with an $\mathrm{IC}_{50}$ in the sub-nanogram per millilitre range, based on a previously reported FACS-based assay with Vero cells. This method is widely used for evaluation of the neutralizing activities of flavivirus monoclonal antibodies ${ }^{22,32}$ and respiratory syncytial virus monoclonal antibodies ${ }^{33}$. However, further studies are needed to investigate the comparability of this method with the standard plaque reduction neutralization test $\left(\mathrm{PRNT}_{80}\right.$ or $\left.\mathrm{PRNT}_{50}\right)$ with overlay, in terms of RVFV infection. Although R5, which targets Gc, showed relatively low biological activity, the antibody still prevents RVFV infection at high concentrations, helping to clear the virus. Accordingly, vaccines containing both Gn and Gc stimulate sera with higher neutralizing activities than vaccines containing Gn alone ${ }^{34}$. 

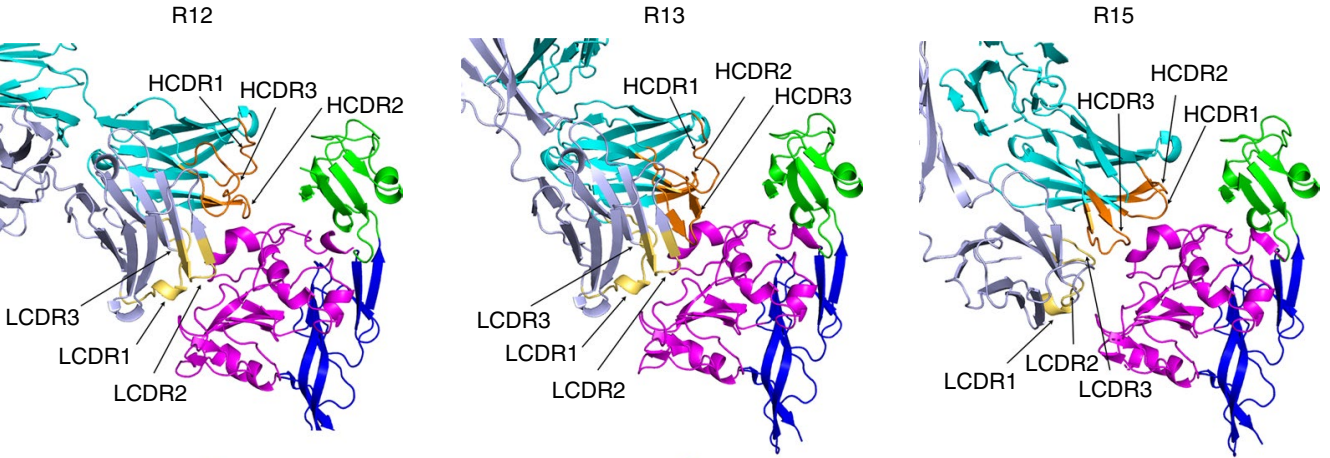

$\mathrm{R} 17$
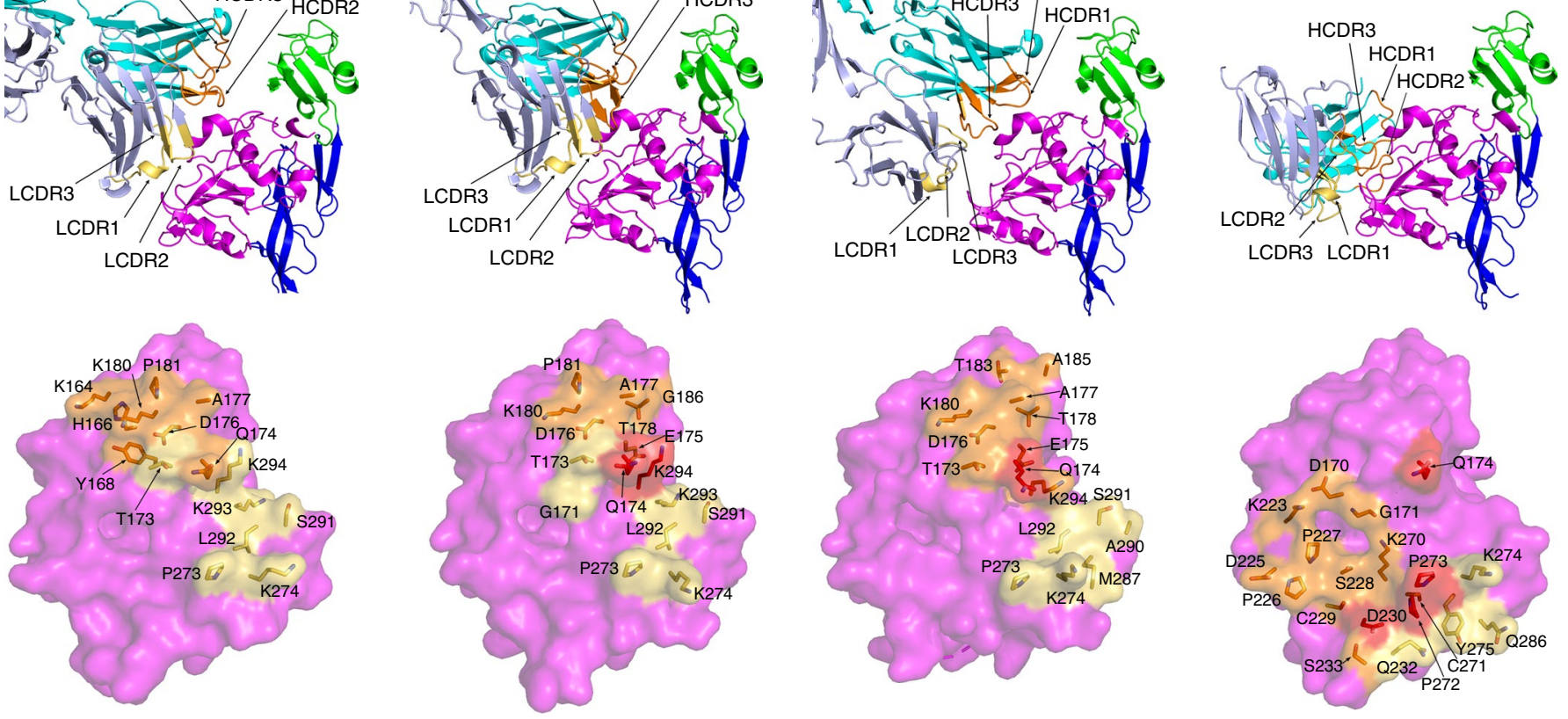

Fig. 4 | Molecular determinants of four human neutralizing Gn monoclonal antibodies. The overall complex structures of $\mathrm{Gn}$ with the indicated monoclonal antibodies are represented in cartoon form in the upper panel. The lower panel delineates the epitope of the indicated monoclonal antibodies on Gn DI. Magenta, blue and green indicate domains I, II and III of RVFV Gn, respectively. For each monoclonal antibody, cyan indicates the heavy chain and light blue represents the light chain. CDRs in the heavy chain are shown in gold. CDRs in the light chain are shown in gold. Accordingly, residues in Gn that form contacts with the heavy or light chains are also highlighted as orange and yellow-orange sticks in the lower panel. The red sticks indicate the residues interacting with both heavy and light chains.

The Gn monoclonal antibodies inhibit the attachment of RVFV virions to the cells in a monoclonal antibody concentration-dependent manner. Interestingly, R15 and R16 seem to have the strongest blocking effects. The blocking effects of R12 and R17 are probably weaker than those of R15 and R16, but stronger than those of R4, R13, R19 and R22, which are consistent with their neutralizing activities.

Through structural analysis of Gn-monoclonal antibody complexes, the monoclonal antibodies R12, R13 and R15 are demonstrated to bind to highly overlapping epitopes. Interestingly, their binding affinity to Gn seems to be correlated with their neutralizing activities. R15, which displays the highest binding affinity to $\mathrm{Gn}$, confers the best neutralization. R13 exhibits the lowest binding affinity with the lowest inhibitory effect. R12 is in the middle. Additionally, the decreased interaction between $\mathrm{R} 12 \mathrm{H}-13 \mathrm{~L}$ also results in a reduced preventive effect, as compared with the parental R12. Thus, for monoclonal antibodies targeting similar epitopes, higher binding affinities are indicated to be linked to better neutralizing activities. Notably, R17 displays the weakest interaction with Gn but inhibits RVFV infection at the nanogram per millilitre level. Further docking of this monoclonal antibody to the RVFV virion indicates approximately $20 \AA$ as the shortest distance between R17 associating with adjacent $\mathrm{Gns}$ without steric hindrance. Based on the reported structures (PDB nos. 1IGY, 1IGT and 1HZH), the distance between two Fabs in one IgG is approximately $40-52 \AA$, suggesting a high possibility that one R17 molecule interacts with more than one Gn molecule on the virion, thereby resulting in high neutralizing activities. Additionally, one R12 or R15 may also bind two Gns. Although the distance between the two most adjacent R13 Fab regions exceeds $60 \AA$, we cannot exclude the possibility that one R 13 binds to two Gns, due to the flexibility of IgG.

Further alanine screening indicates that patch $\mathrm{A}$ is critical for five of the monoclonal antibody interactions (R12, R13, R19, R15 and R16), while patch B is essential for four (R15, R16, R4 and R17). R17 also depends on patch C. Additionally, other residues, including Ser 291, Leu 292 and Lys 293, also contribute to the interaction between $\mathrm{Gn}$ and the monoclonal antibodies. Further studies are still needed to validate the roles of either patches A and B, or other regions, for the neutralization process, by using live RVFV virus possessing the mutations. The isolation of multiple monoclonal antibodies targeting similar regions suggests that these regions are truly hotspot neutralizing targets. There are several strains containing substitutions in individual residues within patches $\mathrm{A}$ and $\mathrm{C}$. These naturally occurring variations could reduce the interaction with all Gn monoclonal antibodies to an extent, highlighting the necessity to identify monoclonal antibodies targeting other epitopes.

A previous report has indicated that DIII also contains neutralizing epitopes, including those targeted by murine monoclonal antibody 4-39-CC ${ }^{35}$. Recently, monoclonal antibody 4-5, an SFTSVspecific neutralizing antibody, was also determined to bind to $\alpha 6$ on SFTSV DIII ${ }^{13}$. Due to the diverse residues within the epitope, monoclonal antibody 4-5 does not interact with RVFV Gn. The RVFV Gn monoclonal antibodies in this study do not bind to SFTSV Gn either. Usually, accessible epitopes on the virions stimulate neutralizing monoclonal antibodies more easily than do cryptic ones. The distribution pattern of neutralizing epitopes on both Phlebovirus Gns supports the model recently proposed for Gn organization ${ }^{13,14}$. The fact that antibodies binding to Gn DI can block the interaction between soluble $\mathrm{Gn}$ protein and host cells implies that Gn DI is probably responsible for receptor binding.

In summary, we isolated eight Gn-specific monoclonal antibodies and one Gc-specific monoclonal antibody from a patient infected with RVFV. The Gn monoclonal antibodies exert high neutralizing activities in vitro and complete protection against RVFV 


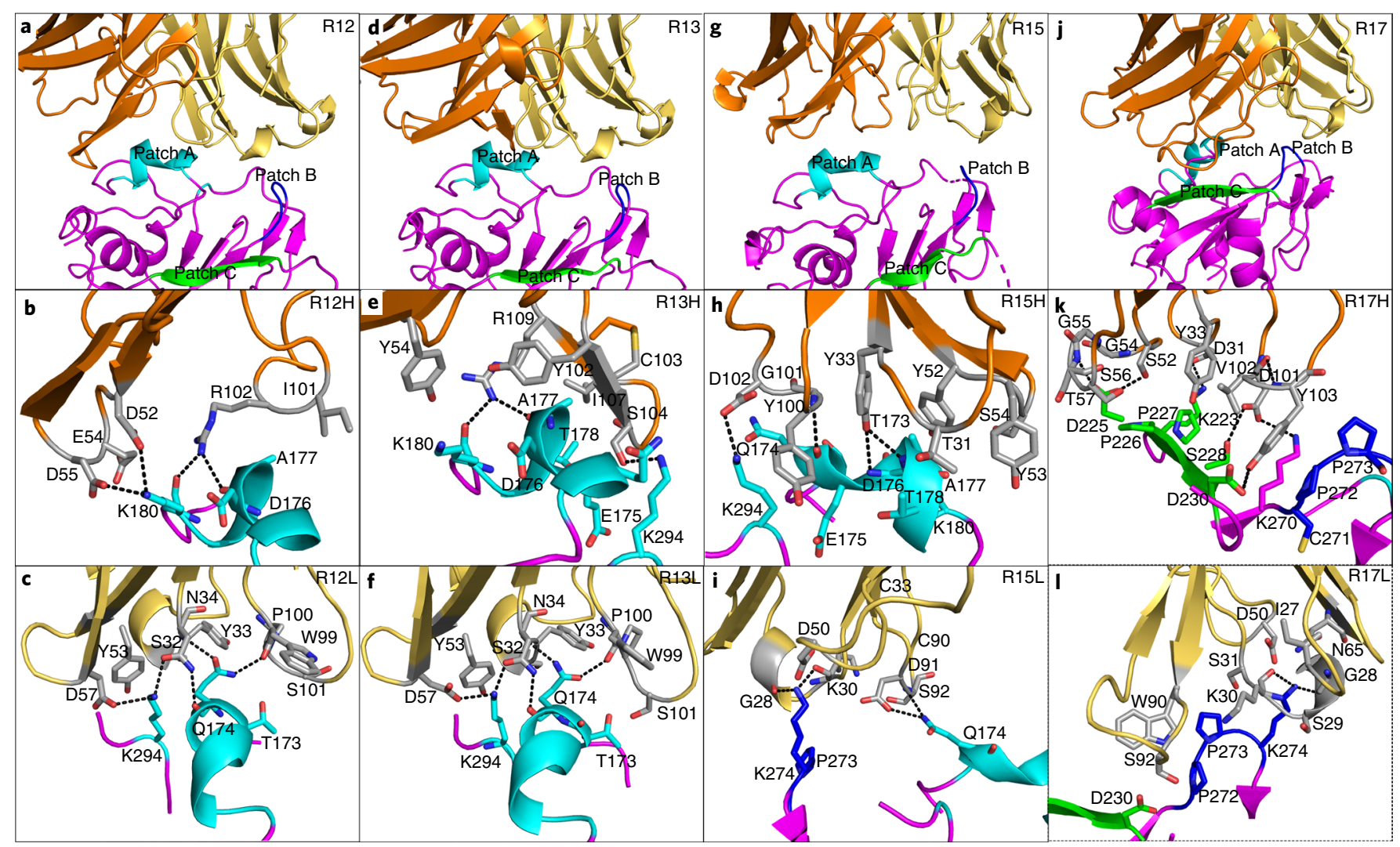

Fig. 5 | The detailed atomic interaction at the $\mathrm{Gn} /$ monoclonal antibody interface. a-I, Cartoon representation of the interface between $\mathrm{Gn}$ and the indicated monoclonal antibody. $\mathrm{Gn}$ DI is marked in magenta. Antigenic site $\mathrm{A}$ is highlighted in cyan, antigenic site $\mathrm{B}$ in blue, and antigenic site $\mathrm{C}$ in green. Orange indicates the heavy chain and yellow-orange represents the light chain. Residues in monoclonal antibodies responsible for the interaction with $\mathrm{Gn}$ are displayed as grey sticks. The figures in the four columns, from left to right, indicate the detailed interaction of Gn with R12, R13, R15 and R17, respectively. In each row, the pictures indicate the interface of $\mathrm{Gn}$ with the indicated monoclonal antibody (the upper panel), and the heavy (the middle panel), and light chains (the lower panel). The black dashed lines suggest the hydrogen bonds probably formed between the indicated atoms.

challenge in vivo, and are worthy of future development as biological therapeutics and prophylaxis.

\section{Methods}

Isolation of antigen-specific single memory $\mathrm{B}$ cells. A patient returning from Angola to China was diagnosed with RVF, representing the first imported case of RVFV infection in China ${ }^{6}$. On day 46 of the disease, blood was taken after written consent using guidelines approved by the ethics committee of the Beijing Ditan Hospital. Peripheral blood mononuclear cells were isolated following the manufacturer's instructions (GE Healthcare) before being subjected to cell sorting as previously reported ${ }^{22,36}$. Briefly, peripheral blood mononuclear cells were incubated with both His-tagged $\mathrm{Gn}$ and His-tagged $\mathrm{Gc}$ at $100 \mathrm{nM}$ before staining with anti$\mathrm{CD} 3$, anti-CD16, anti-CD235a, anti-CD19, anti-CD38, anti-CD27 and anti-His. Antigen-specific memory B cells, which were $\mathrm{CD}^{-}, \mathrm{CD}^{-} 6^{-}, \mathrm{CD} 235 \mathrm{a}^{-}, \mathrm{CD} 38^{-}$, $\mathrm{CD}_{19}^{+}, \mathrm{CD}_{27} 7^{+} \mathrm{hIgG}^{+}$and $\mathrm{His}^{+}$were sorted into 96 -well PCR plates with one cell per well. Flow cytometric analysis and cell sorting were performed on a BD FACSAria III flow cytometer (BD Biosciences) and the data were analysed using FlowJo.

Determination of Ig variable region sequences from sorted single B cells by reverse transcription-PCR. The genes encoding $\operatorname{Ig} V_{H}$ and $V_{L}$ chains were amplified by reverse transcription and nested PCR using an optimized version of a previously reported method, and the primers used were similar to those reported earlier ${ }^{37}$. Briefly, synthesis of Ig $V_{H}$ and $V_{L}$ was performed in 96-well PCR plates containing sorted single $\mathrm{B}$ cells. The reverse transcription reaction was carried out following the manufacturer's instructions (Invitrogen). The primers used for reverse transcription were a mixture, including primers specific for $\operatorname{IgA}, \operatorname{IgD}$, $\operatorname{IgE}$, IgG, IgM, IgK and Ig $\lambda$ constant regions. After complementary DNA synthesis, $V_{H}$, $V_{k}$ and $V_{\lambda}$ genes were amplified separately by two rounds of PCR in 96-well PCR plates. As a template, $2 \mu \mathrm{l}$ of reverse transcription reaction product was used in the first-round of PCR, $1 \mu$ product of which was used to further amplify variable genes in the nested second round of PCR. Samples of $V_{H}, V_{k}$ and $V_{\lambda}$ chain PCR products were analysed on $1.2 \%$ agarose gels. Bands at about 400 bp were cut, purified by the Gel Extraction Micro Kit (CWBIO) and sequenced. Sequences were analysed using the IMGT information system ${ }^{38}$ to identify variable region gene segments and somatic mutations.

Gene construction. The PCR-amplified and sequence-confirmed $V_{H}, V_{k}$ and $\mathrm{V}_{\lambda}$ were separately linked to the indicated constant region by overlapping PCR. These constant regions included the IgG1 constant region (GeneBank Accession no. BC041037) for the heavy chain, the Ig kappa constant region (GeneBank accession no. BC073791) for the $\kappa$ chain and the Ig lambda constant region (GeneBank Accession no. BC073769) for the L fragment. The Ig leader sequence (METDTLLLWVLLLWVPGSTGD) coding region was introduced to the $5^{\prime}$ end of the variable region using primers. The overlapping product containing the leader sequence, variable region and constant region was ligated into the pCAGGS plasmid (Addgene) for monoclonal antibody expression, using EcoRI and XhoI restriction sites for IgG and Ig $\lambda$ cloning, and SacI and XhoI restriction sites for Igk cloning. The coding regions of the $\mathrm{Gn}$ monoclonal antibodies were also constructed in $\mathrm{V}_{\mathrm{L}}-(\mathrm{GGGGS})_{4}-\mathrm{V}_{\mathrm{H}}$ form and cloned into the pET21a expression vector (Invitrogen) through NheI and XhoI restriction sites.

The constructs used for the expression and purification of RVFV and SFTSV glycoproteins were the same as those previously reported ${ }^{12,13}$. Specifically, coding regions for RVFV Gn (M residues 154-469, GenBank accession number: JQ068143.1), RVFV Gc (M residues 691-1119, GenBank accession number: JQ068143.1), SFTSV Gn (M residues 20-452, GenBank accession number: JF906057.1) and SFTSV Gc (M residues 563-996, GenBank accession number: JF906057.1) were individually cloned into the EcoRI and XhoI restriction sites of the pFastBacl vector. For each protein, an N-terminal gp67 signal peptide ${ }^{39}$ and a C-terminal hexa-His were added to facilitate protein secretion and purification.

For Gn tetramer preparation, coding regions for RVFV Gn were cloned into the $\mathrm{KpnI}$ and XhoI restriction sites of pCAGGS. The sequential biotinylation tag and His tag were linked to the $3^{\prime}$ end of Gn by a GS linker.

The constructs of pEGFP-M (M residues 1-1197, GenBank accession number: JQ068143.1), pEGFP-Gn (M residues 154-616, GenBank accession number: JQ068143.1) and pEGFP-Gc (M residues 635-1197, GenBank accession number: 

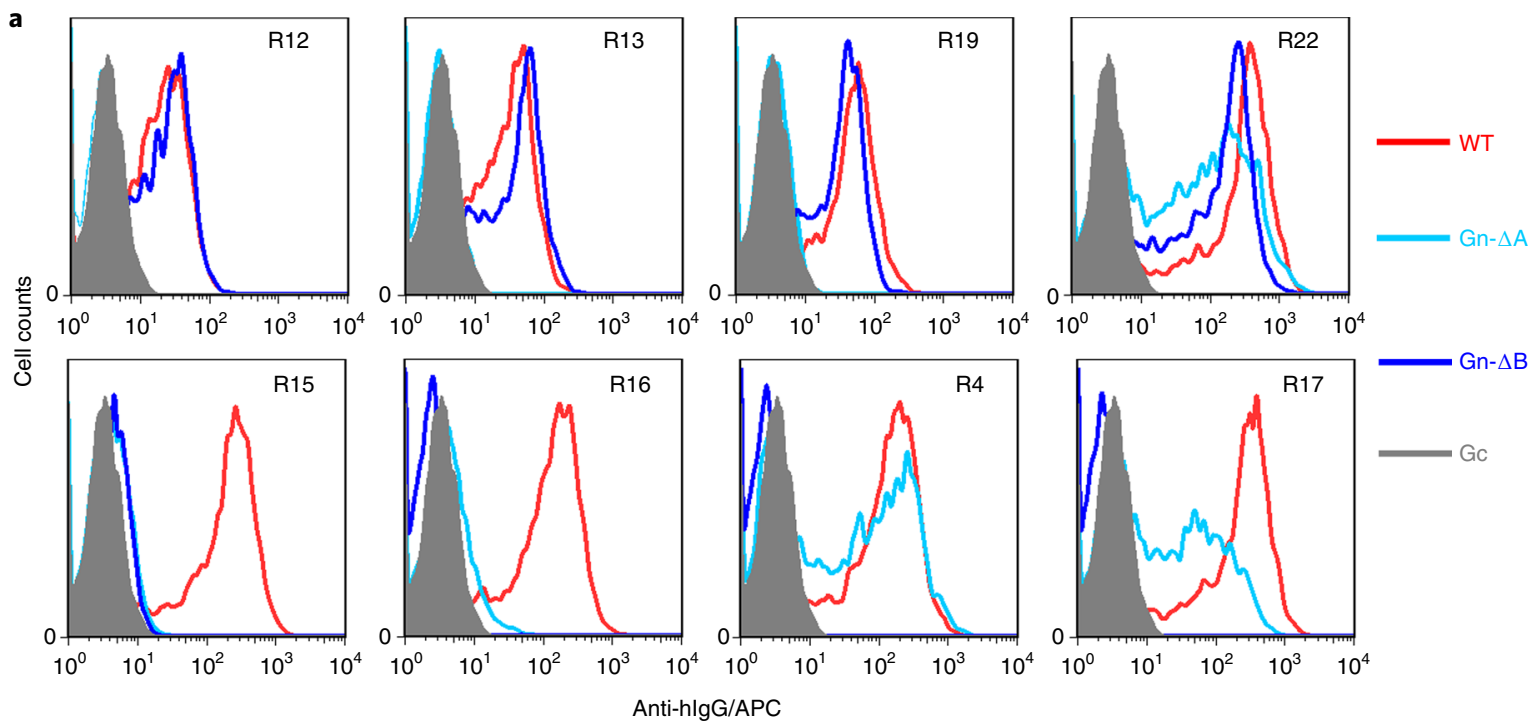

b

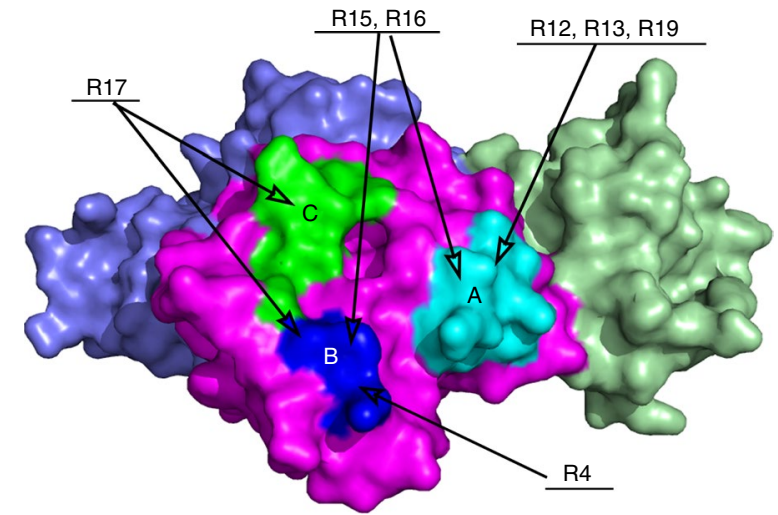

c

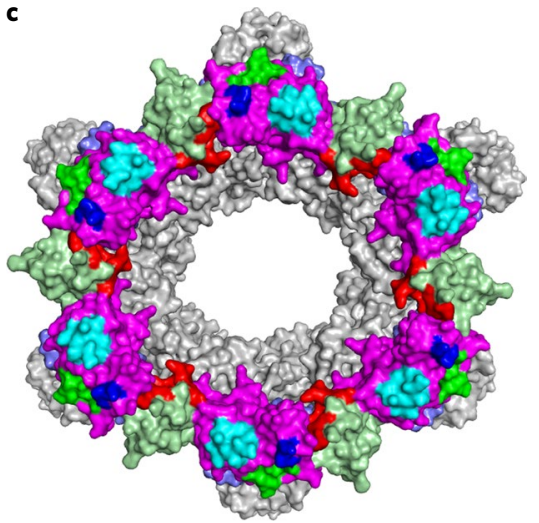

Fig. 6 | Identification of neutralizing hotspots located on Gn DI. a, A flow cytometric assay of the indicated monoclonal antibodies involved in mutated Gn binding. HEK293T cells were transfected with plasmids containing eGFP-fused $G n$ (red), Gn without epitope A (Gn- $\Delta$ A in cyan), Gn without epitope $B(G n-\Delta B$ in blue) and $G c$ (grey), respectively, and were then tested for binding with the indicated monoclonal antibody. Cells transiently expressing $M-$ eGFP stained with secondary antibodies (anti-hlgG/APC) were used as a negative control and are indicated by black filling. The data are representative of two independent experiments. b, Distribution of epitopes A (cyan), B (blue) and C (green) on Gn as a surface representation. Gn monoclonal antibodies are grouped on the basis of their critical targets on $\mathrm{Gn}$ and are highlighted with arrows. c, Antigenic sites docking to the cryogenic electron microscopy

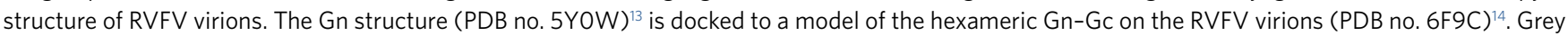
indicates the underlined Gc. Gn DI is displayed in magenta, DII in slate and DIII in pale green. Antigenic sites A, B and C are highlighted in cyan, blue and green, respectively. A previously reported neutralizing epitope located on DIII is shown in red ${ }^{35}$.

JQ068143.1) that were used for FACS were cloned by insertion of the indicated coding regions into pEGFP-N1 at the restriction sites of XhoI and BamHI. Thus, each protein was expressed as a fusion protein with GFP. For pEGFP-Gn and pEGFP-Gc, the MMKVIWFSSLICFVIQCSG sequence was used as a signal peptide and linked to the $5^{\prime}$ end of both genes. Gns incorporating mutations were designed on the basis of the pEGFP-Gn vector. Residues in antigenic patch A ( ${ }_{173}$ TQEDATCK $_{180}$ and Lys 294) were mutated to alanine, except for Cys 179, to maintain the disulfide bond between Cys 179-Cys $188(\mathrm{Gn}-\Delta \mathrm{A})$. P273A and $\mathrm{K} 274 \mathrm{~A}$ were introduced to $\mathrm{Gn}-\Delta \mathrm{B}$ to delete antigenic patch $\mathrm{B}$. Residues in antigenic patch C $\left({ }_{223} \mathrm{KADPPSCD}_{230}\right)$ were also mutated to ${ }_{223} \mathrm{AAAAAACA}_{230}$ $(\mathrm{Gn}-\Delta \mathrm{C})$. We constructed eGFP-fused $\mathrm{Gn}$ mutants that contained single-residue mutations, including Q174A, D176A, K180A, K294A, D225A, S228A or D230A, to investigate the minimum epitopes in patches A and C. T173L, E175G and K294ED230N were also designed on the basis of the PEGFP-Gn vector.

Protein expression and purification. The proteins used for crystallization and SPR experiments were prepared using the Bac-to-Bac baculovirus expression system (Invitrogen). Briefly, recombinant bacmids were prepared and then transfected into sf9 cells to obtain a baculovirus stock, which was then used to infect High 5 cells for protein expression. Target proteins in the supernatant were sequentially collected, affinity-purified by a HisTrap HP column (GE Healthcare) and then subjected to gel filtration (Superdex 200 column, GE Healthcare).
The monoclonal antibodies were expressed in HEK293T cells by transient transfection. Supernatant containing monoclonal antibodies was collected and passed through a HiTrap Protein A FF (1 ml, GE Healthcare) column. The bound protein was detached from the column using $0.1 \mathrm{M}$ sodium citrate, $\mathrm{pH} 4.5$, and further purified by gel filtration (Superdex 200 column, GE Healthcare). For the crystallization study, purified human monoclonal antibodies were digested with the immobilized papain (Thermo Scientific) according to the manufacturer's instructions. The monoclonal antibodies were first buffered in $20 \mathrm{mM} \mathrm{Na}_{3} \mathrm{PO}_{4}$, $10 \mathrm{mM}$ EDTA ( $\mathrm{pH} 7.0$ ), and then digested in buffer containing $20 \mathrm{mM} \mathrm{Na}_{3} \mathrm{PO}_{4}$, $10 \mathrm{mM}$ EDTA, $10 \mu \mathrm{M}$ cysteine- $\mathrm{HCl}(\mathrm{pH} 7.0)$ at $37^{\circ} \mathrm{C}$ overnight. The fragments of Fab were purified by HiTrap Protein A FF ( $5 \mathrm{ml}$, GE Healthcare) and gel filtration (Superdex 200 column, GE Healthcare).

The Gn monoclonal antibody in the scFv form was overexpressed in Escherichia coli as inclusion bodies, and then sequentially dissolved in $8 \mathrm{M}$ urea and diluted against a refolding buffer (100 mM Tris, $\mathrm{pH} 8.0 ; 400 \mathrm{mM}$-arginine; $5 \mathrm{mM}$ EDTA-Na; $5 \mathrm{mM}$ glutathione; $0.5 \mathrm{mM}$ glutathione disulfide). After $12 \mathrm{~h}$ of slow stirring at $4^{\circ} \mathrm{C}$, the refolded scFv was then concentrated and changed to a $20 \mathrm{mM}$ Tris- $\mathrm{HCl}(\mathrm{pH} 8.0)$ and $150 \mathrm{mM} \mathrm{NaCl}$ buffer, and further analysed by gel filtration (Superdex 200 column, GE Healthcare).

Gn with both the biotinylation tag (GLNDIFEAQKIEWHE) and $6 \times$ His tag at the C terminus was expressed in HEK293T cells. After purification by nickel affinity purification (HisTrap HP column, GE Healthcare) and gel filtration 
(Superdex 200 column, GE Healthcare), Gn was biotinylated using the Bir A enzyme (Avidity). Biotin is a small protein that forms a strong bond with streptavidin. PE-tagged streptavidin (BioSource International) was added at a molar ratio of 4:1 to the bioengineered Gn monomers, with the biotin-streptavidin interaction causing four $\mathrm{Gn}$ monomers to bind to streptavidin and create a tetramer. The $\mathrm{Gn}$ tetramers were then used to analyse the interaction between $\mathrm{Gn}$ and cells. MHC tetramers were also prepared as previously reported ${ }^{40}$ and used as a negative control.

Enzyme-linked immunosorbent assay. The binding activities of patient plasma to RVFV Gn and Gc were determined by an enzyme-linked immunosorbent assay. The purified Gn, Gc and Ebola Gp (negative control) were immobilized at $200 \mathrm{ng}$ per well in PBS on 96 -well plates at $4{ }^{\circ} \mathrm{C}$ overnight. After blocking with $1 \%$ BSA at room temperature for $1 \mathrm{~h}$, diluted patient plasma was added and incubated at room temperature for $1 \mathrm{~h}$. The wells were then washed with PBST $(0.5 \%$ Tween 20$)$ for $5 \times 5$ min. Goat anti-human IgG/HRP (Santa Cruz Biotechnology) diluted with PBST at 1:3,000 was added and incubated at room temperature for $1 \mathrm{~h}$, followed by washing with PBST for $5 \times 5 \mathrm{~min}$. TMB $\left(3,3^{\prime}, 5^{\prime}, 5^{\prime}\right.$-tetramethylbenzidine) substrate (CWBio) was added and $2 \mathrm{M} \mathrm{HCl}$ was applied to stop the reaction. Absorbance at $450 \mathrm{~nm}$ was measured and recorded. The bar chart was generated using GraphPad Prism 5.

Flow cytometry. HEK293T cells transiently transfected with plasmids of pEGFP-M, pEGFP-Gn, pEGFP-Gc or pEGFP-Gn mutants (T173L, E175G, K294ED230N, Q174A, D176A, K180A, D225A, S228A, D230A and K294A) were used for the binding test. At $48 \mathrm{~h}$ post-transfection, cells were collected and stained with the indicated purified RVFV monoclonal antibodies at a concentration of $20 \mu \mathrm{g} \mathrm{ml}^{-1}$, except for R12, R13 and Z3L1, which were used at $50 \mu \mathrm{g} \mathrm{ml}^{-1}$. After washing, anti-hIgG/APC was added. The cells were then subjected to analysis using a BD FACSCalibur.

Huh7 cells were incubated with the Gn tetramer at a concentration of $10 \mu \mathrm{g} \mathrm{ml}^{-1}$. For the binding-blocking assay, $\mathrm{Gn}$ tetramers were pre-incubated with the indicated RVFV monoclonal antibody $\left(0.6 \mathrm{mg} \mathrm{m}^{-1}\right)$ before their addition to Huh7 cells. The MHC tetramer was used as a negative control for the Gn tetramer, and Z3L1 was used as a negative control for the RVFV monoclonal antibody. After washing, streptavidin-PE $\left(1 \mu \mathrm{g} \mathrm{m}^{-1}\right)$ was added. Then, the cells were subjected to analysis using a BD FACSCalibur.

Evaluation of the blocking effect of RVFV monoclonal antibody on viral attachment. We performed these experiments using a previously reported method with some modifications ${ }^{27}$. To test the association of antibodies with Huh7 and Vero cells (virus binding setting), both cell lines $\left(2 \times 10^{5}\right)$ were incubated with RVFV virions at $5 \times 10^{4}$ plaque-forming unit (PFUs) at $4{ }^{\circ} \mathrm{C}$ for $30 \mathrm{~min}$. In addition, $2.5 \times 10^{5} \mathrm{PFU}$ RVFV was also used to test the interaction with Huh7 (marked as $5^{*}$ virus in Supplementary Fig. 6). After washing with PBS 3 times, the cells were fixed with $0.5 \%$ paraformaldehyde (PFA) on ice for $30 \mathrm{~min}$, and then washed with PBS twice. Final concentrations of $200 \mu \mathrm{g} \mathrm{ml}^{-1}$ of either Z3L1 (negative control) or the relative RVFV monoclonal antibody were used to stain cells for $30 \mathrm{~min}$ on ice. After washing with PBS three times, anti-hIgG/APC (final concentration of $5 \mu \mathrm{g} \mathrm{ml}^{-1}$, BioLegend) was incubated with cells for $30 \mathrm{~min}$ on ice. Afterwards, the cells were washed with PBS three times and subjected to analysis by flow cytometry.

We used Vero cells to evaluate the blocking effects of RVFV monoclonal antibodies against viral attachment (blocking setting) using a previously published method with some modifications ${ }^{27}$. Virus $\left(5 \times 10^{4} \mathrm{PFUs}\right)$ was pre-incubated with $200 \mu \mathrm{g} \mathrm{ml}^{-1}$ of RVFV monoclonal antibody at $37^{\circ} \mathrm{C}$ for $1 \mathrm{~h}$, and then placed on ice for $30 \mathrm{~min}$. The virus and the indicated monoclonal antibody mixture were added to Vero cells $\left(2 \times 10^{5}\right)$ and placed on ice for another $30 \mathrm{~min}$. After washing with PBS 3 times, the cells were fixed with $0.5 \%$ PFA for $30 \mathrm{~min}$ on ice. Subsequently, the cells were washed with PBS twice and incubated with anti-hIgG/APC (final concentration of $5 \mu \mathrm{g} \mathrm{ml}^{-1}$ ). Finally, the cells were washed with PBS three times and subjected to analysis by flow cytometry (marked as Indicated monoclonal antibody in Fig. 3 b). In parallel, Vero cells $\left(2 \times 10^{5}\right)$ were incubated with cold RVFV $\left(5 \times 10^{4}\right.$ PFUs) for $30 \mathrm{~min}$ on ice. After washing with PBS three times, the cells were subsequently fixed with PFA and stained with the indicated monoclonal antibody at a final concentration of $200 \mu \mathrm{g} \mathrm{ml}^{-1}$ and anti-hIgG/APC (final concentration of $5 \mu \mathrm{g} \mathrm{ml}^{-1}$ ), as depicted in the above paragraph (virus binding setting, marked as Virus binding in Fig. 3b).

The blocking effects of the Gn monoclonal antibodies were further evaluated using serially diluted monoclonal antibodies. In this experiment, the fluorescence of cells was evaluated in both virus binding and blocking settings as depicted above, except that $2.5 \times 10^{4}$ PFUs RVFV were used. Each monoclonal antibody was tested at a final concentration of 100,20, 4 and $0.8 \mu \mathrm{g} \mathrm{ml}^{-1}$. Compared with the sample in the virus binding test, the decreased proportion of hIgG/APC ${ }^{+}$cells in the blocking setting is the inhibitory effect of the indicated monoclonal antibody at the tested concentration.

SPR analysis. The interaction between purified antibodies with RVFV Gn or Gc was monitored by SPR using a BIAcore T100 system (GE Healthcare) carried out at $25^{\circ} \mathrm{C}$ in single-cycle mode. The CM5 biosensor chip (GE Healthcare) was first immobilized with anti-human antibody for flow cells (Fc) 1 and 2, according to the manufacturer's amine-coupling chemistry protocol (GE Healthcare). The indicated monoclonal antibody was then injected and captured on Fc 2. Fc 1 was used as the negative control. Gn or Gc protein was serially diluted and injected. Specifically, both SFTSV Gn and Gc were loaded at concentrations of 50, 100, 200, 400 and $800 \mathrm{nM}$. RVFV Gc was loaded at concentration of 50, 100, 200, 400, 800 and 1,600 $\mathrm{nM}$ for all monoclonal antibodies, except for R5, which was tested at 100, 200, 400, 800 and 1,600 nM. In addition, RVFV Gn at concentrations of 50, 100, 200, 400 and $800 \mathrm{nM}$ was used for R5. The serial dilutions of RVFV Gn used for R13, R16, R17, R12H-13L and R13H-12L were 100, 200, 400, 800 and 1,600 nM; for R4 and R12 were 25, 50, 100, 200 and $400 \mathrm{nM}$; for R19 were 12.5, 25, 50, 100 and $200 \mathrm{nM}$; and for R15 and R22 were $6.25,12.5,25,50$ and $100 \mathrm{nM}$. The binding responses were recorded. All proteins used in the experiment were exchanged into the HBS-EP buffer consisting of $10 \mathrm{mM}$ HEPES, pH 7.4, $150 \mathrm{mM} \mathrm{NaCl}$ and $0.005 \% \mathrm{v} / \mathrm{v}$ Tween 20. The equilibrium dissociation constants (binding affinity, $K_{\mathrm{d}}$ ) for each antigenmonoclonal antibody interaction were calculated using Biacore T100 Evaluation Software, version 1.0 (GE Healthcare). The $K_{\mathrm{d}}$ values for R5 to RVFV Gc and R22 to RVFV Gn were calculated using the model of 1:1 (Langmuir) binding mode, while those for R4, R12, R13, R15, R16, R17, R19, R12H-13L and R13H-12L to Gn were calculated using the model of steady-state affinity.

Neutralization assay by flow cytometry. RVFV was propagated in Vero cells. For the antibody-neutralizing activity of each RVFV monoclonal antibody, a flow cytometry-based neutralization assay with Vero cells was conducted using a similar protocol to that previously reported ${ }^{22}$ with some modifications. Briefly, $2 \times 10^{5}$ cells per well were plated in a 24 -well plate $24 \mathrm{~h}$ before conducting the experiment. The isolated monoclonal antibodies were serially diluted and incubated with RVFV $\left(8 \times 10^{3}\right.$ PFUs). Then, the mixtures were used to infect Vero cells. After $48 \mathrm{~h}$, cells were collected and stained by R4 monoclonal antibody at $2 \mu \mathrm{g} \mathrm{ml}^{-1}$ and anti-hIgG/FITC. After washing, the percentages of Gn-positive cells were measured using a BD FACSCanto II. Sigmoidal neutralization curves were generated using GraphPad Prism 5.

Animal protection. All animal experiments were carried out according to the procedures approved by the Chinese Academy of Sciences and comply with all relevant ethical regulations regarding animal research. To determine the median lethal dose $\left(\mathrm{LD}_{50}\right)$ of RVFV infection to $\mathrm{BALB} / \mathrm{c}$ mice, groups of 5 randomly assigned female mice at 6-8 weeks were challenged intraperitoneally with the indicated PFUs of RVFV. Mice were monitored daily for survival for 14 days postinfection.

In the post-exposure setting, groups of 4 (for the monoclonal antibodies R4, $\mathrm{R} 12, \mathrm{R} 13, \mathrm{R} 16, \mathrm{R} 17, \mathrm{R} 19$ and R22, respectively) or 5 (for the monoclonal antibodies $\mathrm{R} 5$ and R15, respectively) randomly assigned BALB/c mice were challenged intraperitoneally with $1 \times 10^{3} \mathrm{PFU}$ RVFV. On the first day post-infection, a single dose of monoclonal antibody $\left(10 \mathrm{mg} \mathrm{kg}^{-1}\right)$ or PBS (marked as Virus, $n=5$ ) was intraperitoneally administered. The PBS group $(n=4)$, in which mice were administered PBS instead of virus, was also included. In the pre-exposure setting, groups of 4 (for the monoclonal antibodies R4, R12, R13, R16, R17 and R19, respectively) or 5 randomly assigned mice (for the monoclonal antibodies R5, R15 and R22, respectively) were injected with either monoclonal antibodies $\left(10 \mathrm{mg} \mathrm{kg}^{-1}\right)$ or PBS $(n=4)$, followed by administration of $1 \times 10^{3}$ PFU RVFV, 1 day later. Mice were monitored daily for survival for 14 days post-infection. Mice exhibiting weight loss of over $20 \%$ of their initial weight on day 0 were euthanized.

Crystallization, data collection and structure determination. The indicated Fabs or scFvs were mixed with $\mathrm{Gn}$ at a stoichiometry of 1:1 and incubated on ice for $2 \mathrm{~h}$. The mixture was then subjected to gel filtration. The eluted peak containing the complex of the indicated Fab and $\mathrm{Gn}$ were pooled, buffered in $20 \mathrm{mM}$ Tris and $50 \mathrm{mM} \mathrm{NaCl}$ (pH 8.0), and concentrated to $10 \mathrm{mg} \mathrm{ml}^{-1}$. Crystallization trials were performed by sitting-drop $(300 \mathrm{nl}+300 \mathrm{nl})$ vapour diffusion at $18^{\circ} \mathrm{C}$. Diffractable crystals of the complex were finally obtained in a solution consisting of $0.2 \mathrm{M}$ magnesium chloride hexahydrate, 0.1 M HEPES sodium, $\mathrm{pH} 7.5$, and $30 \% \mathrm{v} / \mathrm{v} 2$-propanol, with a protein concentration of $10 \mathrm{mg} \mathrm{ml}^{-1}$ for $\mathrm{Gn}-\mathrm{R} 12$ Fab; a solution containing $0.1 \mathrm{M}$ bicine, $\mathrm{pH} 9.0$, and 20\% w/v PEG 6000, with a protein concentration of $10 \mathrm{mg} \mathrm{ml}^{-1}$ for $\mathrm{Gn}-\mathrm{R} 13 \mathrm{Fab}$; a solution containing $0.1 \mathrm{M}$ amino acids, $0.1 \mathrm{M}$ imidazole, MES, pH 6.5, and 30\% w/v P550MME_P20K, with a protein concentration of $10 \mathrm{mg} \mathrm{ml}^{-1}$ for $\mathrm{Gn}-\mathrm{R} 15 \mathrm{Fab}$; a solution containing $0.2 \mathrm{M}$ ammonium sulfate, $0.1 \mathrm{M}$ Tris, $\mathrm{pH} 7.5$, and 20\% w/v PEG $5000 \mathrm{MME}$, with a protein concentration of $15 \mathrm{mg} \mathrm{ml}^{-1}$ for $\mathrm{Gn}-\mathrm{R} 17 \mathrm{scFv}$. Diffraction data were collected at $100 \mathrm{~K}$ at Shanghai Synchrotron Radiation Facility (SSRF) BL19U1. For data collection, the crystals were cryo-protected by briefly soaking in reservoir solution supplemented with $20 \%$ (v/v) glycerol before flash-cooling in liquid nitrogen. The wavelengths used to collect the data for Gn-R12 Fab, Gn-R13 Fab, Gn-R15 Fab and Gn-R17 scFv were $0.97776 \AA 0.97853 \AA, 0.97915 \AA$ and $0.97853 \AA$, respectively. The datasets were processed with HKL2000 software ${ }^{41}$.

The complex structures were determined by the molecular replacement method using Phaser ${ }^{42}$ with previously reported structures of RVFV Gn (PDB code: $5 \mathrm{YOW}$ ) and Fab (PDB code: $1 \mathrm{HZH})$. The atomic models were completed 
with $\operatorname{Coot}^{43}$ and refined with phenix.refine in Phenix ${ }^{44}$, and the stereochemical qualities of the final models were assessed with MolProbity ${ }^{45}$. Of all the residues, a total of $96.59,97.32,95.96$ and $94.94 \%$ were located in the favoured regions of the Ramachandran plot for Gn-R12 Fab, Gn-R13 Fab, Gn-R15 Fab and Gn-R17 $\mathrm{scFv}$, respectively. The outlier regions were determined to be $0.16,0.14,0.00$ and $0.19 \%$ for Gn-R12 Fab, Gn-R13 Fab, Gn-R15 Fab and Gn-R17 scFv, respectively. The data collection and structure refinement statistics are summarized in Supplementary Table 3. The structural figures were generated using Pymol.

Western blotting. To test whether the isolated monoclonal antibodies could recognize the linearized epitope, each monoclonal antibody was subjected to western blotting. We loaded $0.1 \mu \mathrm{g}$ of both purified RVFV Gn and Gc for SDSpolyacrylamide gel electrophoresis, then transferred the proteins to nitrocellulose membranes and incubated them with the identified monoclonal antibodies at a final concentration of $1 \mu \mathrm{g} \mathrm{ml}^{-1}$. A secondary antibody of anti-hIgG/HRP was used at a final concentration of $0.1 \mu \mathrm{g} \mathrm{ml}^{-1}$ (Zhongshanjinqiao Corp.). Directly HRPconjugated anti-His antibodies were used at a final concentration of $0.1 \mu \mathrm{g} \mathrm{ml}^{-1}$ (CWBio). Then, the TMB substrate (CWBio) was added.

Sequence alignments. The GenBank accession number of the sequences used for analysing the conservation of epitopes among Phlebovirus are as follows: Heartland virus, AIF75092.1; Candiru virus, YP_004347992.1; Punta Toro virus, AAA47110.1; Chagres virus, AEL29641.1; sandfly fever Naples virus, AIS25027.1; Toscana virus, ABZ85665.1; Uukuniemi virus, NP_941979; Bhanja virus, YP_009141014.1; and Palma virus, AGC60100.1. The 108 sequences of the RVFV strains, with their complete genomes stored in the NCBI Viral Genomes Resource, are as follows: P03518.2, ABD38806.1, AEB20462.1, ABD38803.1, ABD38804.1, ABD38805.1, ABD38800.1, ABD38807.1, AEF79993.1, ABD38802.1, AJA32703.1, ARW71759.1, ABD38801.1, ABD38808.1, ABD38835.1, AMD08950.1, ABD38798.1, ABD38799.1, ABD38797.1, ABD38796.1, ABD38823.1, ABD38821.1, ABD38820.1, YP 003848705.1, ABD38819.1, ABD38812.1, ABD38815.1, ABD38813.1, ABD38814.1, ABD38817.1, ABD38822.1, ABD38818.1, ABD38816.1, ABD38809.1, ABD38810.1, ABD38811.1, AEC14287.1, AEC14288.1, AGB13833.1, ACE78370.1, ACE78388.1, ACE78379.1, AGB13831.1, AGB13830.1, AGB13832.1, AGB13834.1, ACE78394.1, ACE78383.1, AJQ19496.1, ABD38830.1, ABD38829.1, ABD38828.1, ABD38825.1, ABD38824.1, ABD38826.1, ABD38834.1, ABD38827.1, AJQ19492.1, ABD38831.1, ABD38833.1, ABD38832.1, AEB20468.1, AEB20467.1, AEB20463.1, AEB20472.1, AEB20466.1, AEB20465.1, AEB20464.1, AEC14283.1, ACE78377.1, ACE78376.1, AEC14290.1, ACE78378.1, ACE78382.1, ACE78384.1, ACE78375.1, ACE78386.1, ACE78387.1, ACE78385.1, ACE78381.1, AEB20469.1, AEZ53084.1, AEZ53085.1, CCG28447.1, CCG28444.1, AEZ53088.1, AEZ53087.1, AEZ53086.1, AEL31325.1, AEE10383.1, AEC14291.1, AEC14289.1, AEC14286.1, AEC14285.1, AEC14284.1, AEB20471.1, AEB20470.1, ACE78395.1, ACE78393.1, ACE78392.1, ACE78391.1, ACE78390.1, ACE78389.1, ACE78380.1, ACE78374.1, ACE78373.1, ACE78372.1 and ACE78371.1. The sequence logos were generated using the web server of Weblogo (http://weblogo.berkeley.edu/logo.cgi).

Reporting Summary. Further information on research design is available in the Nature Research Reporting Summary linked to this article.

\section{Data availability}

The crystal structures of Gn complexed with the Fab form of the monoclonal antibodies R12, R13 and R15 and the scFv of R17 have been deposited in the Protein Data Bank under accession codes 6IEK, 6IEA, 6IEB and 6IEC, respectively. The data that support the findings of this study are available from the corresponding author upon request.

Received: 14 September 2018; Accepted: 14 February 2019; Published online: 1 April 2019

\section{References}

1. Daubney, R., Hudson, J. R. \& Garnham, P. C. Enzootic hepatitis or Rift Valley fever. An undescribed virus disease of sheep cattle and man from East Africa. J. Pathol. Bacteriol. 34, 545-579 (1931).

2. Nanyingi, M. O. et al. A systematic review of Rift Valley Fever epidemiology 1931-2014. Infect. Ecol. Epidemiol. 5, 28024 (2015).

3. Rift Valley Fever (RVF). World Organisation for Animal Health http://www. oie.int/en/animal-health-in-the-world/animal-diseases/rift-valley-fever (2019).

4. Rift Valley Fever. World Health Organization http://www.who.int/csr/don/ archive/disease/rift_valley_fever/en/ (2018).

5. Shoemaker, T. et al. Genetic analysis of viruses associated with emergence of Rift Valley fever in Saudi Arabia and Yemen, 2000-01. Emerg. Infect. Dis. 8, 1415-1420 (2002).

6. Liu, J. et al. The first imported case of Rift Valley fever in China reveals a genetic reassortment of different viral lineages. Emerg. Microbes Infect. 6, e4 (2017).

7. Mansfield, K. L. et al. Rift Valley fever virus: A review of diagnosis and vaccination, and implications for emergence in Europe. Vaccine 33, 5520-5531 (2015).
8. Adams, M. J. et al. Changes to taxonomy and the International Code of Virus Classification and Nomenclature ratified by the International Committee on Taxonomy of Viruses (2017). Arch. Virol. 162, 2505-2538 (2017).

9. Knipe, D. M. \& Howley, P. M. Fields Virology 6th edn (Wolters Kluwer/ Lippincott Williams \& Wilkins Health, 2013)

10. de Boer, S. M. et al. Acid-activated structural reorganization of the Rift Valley fever virus Gc fusion protein. J. Virol. 86, 13642-13652 (2012).

11. Dessau, M. \& Modis, Y. Crystal structure of glycoprotein C from Rift Valley fever virus. Proc. Natl Acad. Sci. USA 110, 1696-1701 (2013).

12. Zhu, Y. et al. The post-fusion structure of the Heartland virus Gc glycoprotein supports taxonomic separation of the bunyaviral families Phenuiviridae and Hantaviridae. J. Virol. 92, e01558-01517 (2017).

13. Wu, Y. et al. Structures of phlebovirus glycoprotein $\mathrm{Gn}$ and identification of a neutralizing antibody epitope. Proc. Natl Acad. Sci. USA 114, E7564-E7573 (2017).

14. Halldorsson, S. et al. Shielding and activation of a viral membrane fusion protein. Nat. Commun. 9, 349 (2018).

15. Huiskonen, J. T., Overby, A. K., Weber, F. \& Grunewald, K. Electron cryo-microscopy and single-particle averaging of Rift Valley fever virus: evidence for GN-GC glycoprotein heterodimers. J. Virol. 83, 3762-3769 (2009).

16. Lagerqvist, $\mathrm{N}$. et al. Characterisation of immune responses and protective efficacy in mice after immunisation with Rift Valley Fever virus cDNA constructs. Virol. J. 6, 6 (2009).

17. de Boer, S. M. et al. Rift Valley fever virus subunit vaccines confer complete protection against a lethal virus challenge. Vaccine 28, 2330-2339 (2010).

18. Wallace, D. B. et al. Protective immune responses induced by different recombinant vaccine regimes to Rift Valley fever. Vaccine 24, 7181-7189 (2006).

19. Faburay, B. et al. A recombinant Rift Valley fever virus glycoprotein subunit vaccine confers full protection against Rift Valley fever challenge in sheep. Sci. Rep. 6, 27719 (2016).

20. Papin, J. F. et al. Recombinant Rift Valley fever vaccines induce protective levels of antibody in baboons and resistance to lethal challenge in mice. Proc. Natl Acad. Sci. USA 108, 14926-14931 (2011).

21. Heise, M. T. et al. An alphavirus replicon-derived candidate vaccine against Rift Valley fever virus. Epidemiol. Infect. 137, 1309-1318 (2009).

22. Wang, Q. et al. Molecular determinants of human neutralizing antibodies isolated from a patient infected with Zika virus. Sci. Transl. Med. 8, 369 ra179 (2016)

23. Smith, D. R. et al. The pathogenesis of Rift Valley fever virus in the mouse model. Virology 407, 256-267 (2010).

24. Gao, G. F. From "A"IV to "Z"IKV: Attacks from emerging and re-emerging pathogens. Cell 172, 1157-1159 (2018).

25. Shi, Y., Wu, Y., Zhang, W., Qi, J. \& Gao, G. F. Enabling the 'host jump': structural determinants of receptor-binding specificity in influenza A viruses. Nat. Rev. Microbiol. 12, 822-831 (2014).

26. Hofmann, H. et al. Severe fever with thrombocytopenia virus glycoproteins are targeted by neutralizing antibodies and can use DC-SIGN as a receptor for $\mathrm{pH}$-dependent entry into human and animal cell lines. J. Virol. 87, 4384-4394 (2013).

27. Yamashita, T. et al. Biological and immunological characteristics of hepatitis E virus-like particles based on the crystal structure. Proc. Natl Acad. Sci. USA 106, 12986-12991 (2009).

28. Nderitu, L. et al. Sequential Rift Valley fever outbreaks in eastern Africa caused by multiple lineages of the virus. J. Infect. Dis. 203, 655-665 (2011).

29. Bird, B. H., Khristova, M. L., Rollin, P. E., Ksiazek, T. G. \& Nichol, S. T. Complete genome analysis of 33 ecologically and biologically diverse Rift Valley fever virus strains reveals widespread virus movement and low genetic diversity due to recent common ancestry. J. Virol. 81, 2805-2816 (2007).

30. Frank, A. L., Six, H. R. \& Marchini, A. Human monoclonal antibodies to influenza virus: IgG subclass and light chain distribution. Viral Immunol. 2, 31-36 (1989).

31. Kirchenbaum, G. A., Allen, J. D., Layman, T. S., Sautto, G. A. \& Ross, T. M Infection of ferrets with influenza virus elicits a light chain-biased antibody response against hemagglutinin. J. Immunol. 199, 3798-3807 (2017).

32. Kraus, A. A., Messer, W., Haymore, L. B. \& De Silva, A. M. Comparison of plaque- and flow cytometry-based methods for measuring dengue virus neutralization. J. Clin. Microbiol. 45, 3777-3780 (2007).

33. Chen, M. et al. A flow cytometry-based assay to assess RSV-specific neutralizing antibody is reproducible, efficient and accurate. J. Immunol. Methods 362, 180-184 (2010).

34. Schmaljohn, C. S. et al. Baculovirus expression of the M genome segment of Rift Valley fever virus and examination of antigenic and immunogenic properties of the expressed proteins. Virology 170, 184-192 (1989).

35. Keegan, K. \& Collett, M. S. Use of bacterial expression cloning to define the amino-acid-sequences of antigenic determinants on the G2-glycoprotein of Rift-Valley fever virus. J. Virol. 58, 263-270 (1986).

36. Zhang, Q. et al. Potent neutralizing monoclonal antibodies against Ebola virus infection. Sci. Rep. 6, 25856 (2016). 
37. Liao, H. X. et al. High-throughput isolation of immunoglobulin genes from single human B cells and expression as monoclonal antibodies. J. Virol. Methods 158, 171-179 (2009).

38. Ehrenmann, F., Kaas, Q. \& Lefranc, M. P. IMGT/3Dstructure-DB and IMGT/ DomainGapAlign: a database and a tool for immunoglobulins or antibodies, T cell receptors, MHC, IgSF and MhcSF. Nucleic Acids Res. 38, D301-D307 (2010).

39. Zhang, W. et al. Crystal structure of the swine-origin A (H1N1)-2009 influenza A virus hemagglutinin (HA) reveals similar antigenicity to that of the 1918 pandemic virus. Protein Cell 1, 459-467 (2010).

40. Zhou, M. et al. Screening and identification of severe acute respiratory syndrome-associated coronavirus-specific CTL epitopes. J. Immunol. 177, 2138-2145 (2006)

41. Otwinowski, Z. \& Minor, W. Processing of X-ray diffraction data collected in oscillation mode. Methods Enzymol. 276, 307-326 (1997).

42. Read, R. J. Pushing the boundaries of molecular replacement with maximum likelihood. Acta Crystallogr. D 57, 1373-1382 (2001).

43. Emsley, P. \& Cowtan, K. Coot: model-building tools for molecular graphics. Acta Crystallogr. D 60, 2126-2132 (2004).

44. Adams, P. D. et al. PHENIX: a comprehensive Python-based system for macromolecular structure solution. Acta Crystallogr. D 66, 213-221 (2010).

45. Chen, V. B. et al. MolProbity: all-atom structure validation for macromolecular crystallography. Acta Crystallogr. D 66, 12-21 (2010).

\section{Acknowledgements}

We thank G. Salazar (University of Texas Health Science Center at Houston) for her critical editing of the manuscript. We acknowledge L. Zhang and Q. Zhang (Comprehensive AIDS Research Center, and Collaborative Innovation Center for Diagnosis and Treatment of Infectious Diseases, School of Medicine, Tsinghua University) for their instruction and help with isolating single B cells. We thank the staff of the BL19U1 beamline at Shanghai Synchrotron Radiation Facility (Shanghai, People's Republic of China) for assistance during data collection. We are grateful to J. Jia (Institute of Biophysics, Chinese Academy of Sciences) for technical support of BDAria II manipulation and Y. Chen and Z. Yang (Institute of Biophysics, Chinese Academy of Sciences) for technical help with BIAcore experiments. This work was supported by the Strategic Priority Research Program of the Chinese Academy of Sciences (grant no. XDB29040201), the National Science and Technology Major Projects for 'Major New Drugs Innovation and Development' (grant no. 2018ZX09711003-002-001), the National Science and Technology Major Project (grant nos. 2016ZX10004222008 and 2018ZX10101004-001), the External Cooperation Program of CAS (153211KYSB20160001) and the National Natural Science Foundation of China (NSFC, grant nos. 31872745,31502078 and 81502972 ). Y.S. is supported by the National Science and Technology Major Project (grant no. 2018ZX10101004-001). G.F.G. is also supported by the External Cooperation Program of CAS (grant no. 153211KYSB20160001). Q.W. is supported by Young Elite Scientist Sponsorship Program by China Association for Science and Technology (grant no. 2015QNRC001), the Youth Innovation Promotion Association CAS (grant no. 2018119) and the grant from China Scholarship Council (grant no. 201704910327). Y.W. is supported by the Youth Innovation Promotion Association CAS (grant no. 2016086). J.Y. and G.F.G. are supported by the foundation of the NSFC Innovative Research Group (grant no. 81621091).

\section{Author contributions}

Q.W., Z.T., G.F.G. and J.Y. initiated and coordinated the project. Q.W., Y.W., Z.T., G.F.G. and J.Y. designed the experiments. Q.W., T.M., Y.W., Z.T. and H.Y. conducted the experiments with the assistance of Z.Z., L.W., R.S. and M.Y. F.G., J.Q. and Y.C. collected the crystallographic data and solved the structures. Z.C. and H.Z. coordinated the isolation of peripheral blood mononuclear cells from the RVFV patient. M.L., C.Q., G.F.G. and J.Y. coordinated the viral experiments. T.M. and G.W. manipulated the viruses with the help of Y.B., J.S. and H.J. Q.W., Y.W., Z.T., Z.A., Y.S., G.F.G. and J.Y. analysed the data. Q.W. wrote the manuscript. Y.W., G.W., Z.A., J.W., T.D.Y., Y.S., W.J.L., G.F.G. and J.Y. helped revise the manuscript.

\section{Competing interests}

The authors declare no competing interests.

\section{Additional information}

Supplementary information is available for this paper at https://doi.org/10.1038/ s41564-019-0411-z.

Reprints and permissions information is available at www.nature.com/reprints. Correspondence and requests for materials should be addressed to G.F.G. or J.Y. Publisher's note: Springer Nature remains neutral with regard to jurisdictional claims in published maps and institutional affiliations.

(c) The Author(s), under exclusive licence to Springer Nature Limited 2019 


\section{Reporting Summary}

Nature Research wishes to improve the reproducibility of the work that we publish. This form provides structure for consistency and transparency in reporting. For further information on Nature Research policies, see Authors \& Referees and the Editorial Policy Checklist.

\section{Statistical parameters}

When statistical analyses are reported, confirm that the following items are present in the relevant location (e.g. figure legend, table legend, main text, or Methods section).

$\mathrm{n} / \mathrm{a} \mid$ Confirmed

$\square$ The exact sample size $(n)$ for each experimental group/condition, given as a discrete number and unit of measurement

$\square$ An indication of whether measurements were taken from distinct samples or whether the same sample was measured repeatedly

$\bigotimes \square$ The statistical test(s) used AND whether they are one- or two-sided

Xnly common tests should be described solely by name; describe more complex techniques in the Methods section.

Х $\square$ A description of all covariates tested

Х $\square$ A description of any assumptions or corrections, such as tests of normality and adjustment for multiple comparisons

$\square$ A full description of the statistics including central tendency (e.g. means) or other basic estimates (e.g. regression coefficient) AND

A full description of the statistics including central tendency (e.g. means) or other basic estimates (e.
variation (e.g. standard deviation) or associated estimates of uncertainty (e.g. confidence intervals)

$\bigotimes$ For null hypothesis testing, the test statistic (e.g. $F, t, r$ ) with confidence intervals, effect sizes, degrees of freedom and $P$ value noted

Х $\square$ Give $P$ values as exact values whenever suitable.

Х $\square$ For Bayesian analysis, information on the choice of priors and Markov chain Monte Carlo settings

Х $\square$ For hierarchical and complex designs, identification of the appropriate level for tests and full reporting of outcomes

\ $\square$ Estimates of effect sizes (e.g. Cohen's $d$, Pearson's $r$ ), indicating how they were calculated

$\varnothing$ Clearly defined error bars

State explicitly what error bars represent (e.g. SD, SE, CI)

Our web collection on statistics for biologists may be useful.

\section{Software and code}

Policy information about availability of computer code

Data collection Not applicable to this study, no algorithms or software not previously described was used.

Data analysis Not applicable to this study.

For manuscripts utilizing custom algorithms or software that are central to the research but not yet described in published literature, software must be made available to editors/reviewers upon request. We strongly encourage code deposition in a community repository (e.g. GitHub). See the Nature Research guidelines for submitting code \& software for further information.

\section{Data}

Policy information about availability of data

All manuscripts must include a data availability statement. This statement should provide the following information, where applicable:

- Accession codes, unique identifiers, or web links for publicly available datasets

- A list of figures that have associated raw data

- A description of any restrictions on data availability

There are no restrictions on data availability. 


\section{Field-specific reporting}

Please select the best fit for your research. If you are not sure, read the appropriate sections before making your selection.

$\bigotimes$ Life sciences $\quad \square$ Behavioural \& social sciences $\square$ Ecological, evolutionary \& environmental sciences

For a reference copy of the document with all sections, see nature.com/authors/policies/ReportingSummary-flat.pdf

\section{Life sciences study design}

All studies must disclose on these points even when the disclosure is negative.

Sample size $\quad$ For mice experiments, the study is powered to confirm a difference in survival of at least $100 \%$ at the $95 \%$ confidence interval with $80 \%$ power, as calculated with the following website from the UCSF Clinical \& Translational Science Institute: http://www.sample-size.net/samplesize-proportions/. For the samples in flow cytometry, experiments were performed in duplicate or triplicate, as indicated in the Materials and Methods, and Figure legends.

Data exclusions No data were excluded.

Replication The mice experiment was not repeated due to robust findings in the animal experiments (with different antibodies) and supporting structural data. Cell staining assays were performed in duplicate or triplicate, as indicated in the Materials and Methods, and Figure legends.

Randomization The animals used in this study were allocated by random individual numbering into experimental groups (i.e. A1, A2, B1, B2...etc.)

Blinding The investigators were not blinded to the study.

\section{Reporting for specific materials, systems and methods}

Materials \& experimental systems

Involved in the study
$\square$ Unique biological materials

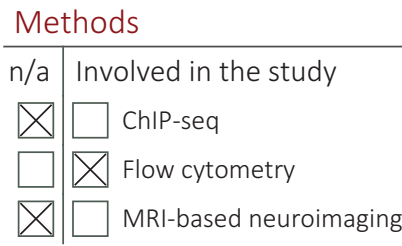

\section{Antibodies}

Antibodies used

Validation

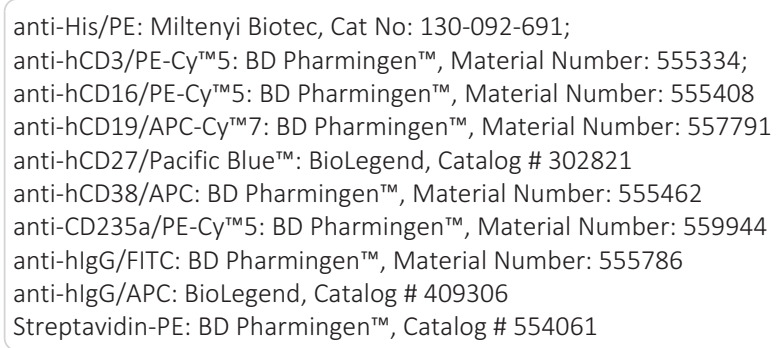
We follow the manufactures' instruction to use the above listed antibodies to stain human PBMCs. All antibodies work well.

\section{Eukaryotic cell lines}

Policy information about cell lines

Cell line source(s)

ATCC

Authentication

The cell lines were not authenticated since they were purchased commercially and are not commonly misidentified. 
Mycoplasma contamination

Commonly misidentified lines (See ICLAC register)
The cells were not tested for mycoplasma contamination.

The cell lines used in this study do not appear on the ICLAC register.

\section{Animals and other organisms}

Policy information about studies involving animals; ARRIVE guidelines recommended for reporting animal research

Laboratory animals

BALB/c, female, 6-8 weeks old

Wild animals

Field-collected samples
Not applicable to this study.

Not applicable to this study.

\section{Human research participants}

Policy information about studies involving human research participants

Population characteristics

We used the blood of the first imported Rift Valley fever case in China. The patient was 45 -year-old man when the biospecimen was collected.

Recruitment

On Day 7 of the disease onset, the patient returned from Angola to Beijing and was admitted to Beijing Ditan Hospital. Upon admission to the hospital, the patient agreed to provide the biospecimen for detection, further diagnostic and scientific research.

\section{Flow Cytometry}

Plots

Confirm that:

\The axis labels state the marker and fluorochrome used (e.g. CD4-FITC).

\The axis scales are clearly visible. Include numbers along axes only for bottom left plot of group (a 'group' is an analysis of identical markers).

$\bigotimes$ All plots are contour plots with outliers or pseudocolor plots.

$\bigotimes$ A numerical value for number of cells or percentage (with statistics) is provided.

\section{Methodology}

Sample preparation

Instrument

Software

Cell population abundance

Gating strategy

\section{Sample preparation listed in Methods}

BD FACSAria II, BD FACSCalibur and BD FACSCanto II.

\section{FlowJo}

After cell sorting, the collected cells were subjected for RT-PCR to determine the genes of the IgG. Thus, we did not test the relevant cell populations within post-sort fractions.

For the isolation of antigen-specific memory B cells, we used the unstained PBMCs as control to gate cells. In order to evaluate the binding of indicated MAbs to the specific antigen, cells that expressed M genes and were stained with anti-hlgG/APC, were used as the control for gating.

In order to evaluate the effectiveness of the indicated MAb on preventing the binding of Gn tetramers to Huh7, Huh7 cells that were sequentially incubated with MHC-tetramer and streptavidin-PE were used as the control to gate cells.

To investigate the interaction of Gn MAbs with RVFV, Vero or Huh 7 cells incubated with RVFV and then sequentially fixed with PFA, stained with Z3L1 (MAb against Zika, used as negative control) and anti-hlgG/APC were used as control to gate cells.

To test the blocking effect of RVFV MAb on viral attachment, cells stained with indicated MAb and anti-hlgG/APC used as control to gate the cell.

To evaluate the interaction between Gn MAbs and Gn mutants, HEK293T cells, which transiently expressed GFP-tagged Gn mutants, stained with Z3L1 used as control to gate cells.

To map the critical patch for indicated MAbs binding, HEK293T cells, which transiently expressed RVFV-Gc-eGFP and stained with indicated MAb as control to gate the cell.

\Tick this box to confirm that a figure exemplifying the gating strategy is provided in the Supplementary Information. 